\title{
Multiplexed live-cell visualization of endogenous proteins with nanometer precision by fluorobodies
}

${ }^{1}$ Institute of Biochemistry, Biocenter, Goethe University Frankfurt, Max-von-Laue Str. 9, 60438

8 Frankfurt/Main, Germany; ${ }^{2}$ Institute of Physical and Theoretical Chemistry, Goethe University

9 Frankfurt, Max-von-Laue Str. 7, 60438 Frankfurt/Main, Germany; ${ }^{3}$ Cluster of Excellence -

10 Macromolecular Complexes, Goethe University Frankfurt, Max-von-Laue Str. 9, 60438

11 Frankfurt/Main, Germany

$13{ }^{*}$ Correspondence should be addressed to R.W. (wieneke@em.uni-frankfurt.de) or

R.T. (tampe@em.uni-frankfurt.de)

Key words: endogenous protein labeling, in vivo labeling, live-cell imaging, nanobody, 


\section{Abstract}

2 The visualization of endogenous proteins in living cells is a major challenge. A fundamental

3 requirement for spatiotemporally precise imaging is a minimal disturbance of protein function at

4 high signal-to-background ratio. Current approaches for visualization of native proteins in living

5 cells are limited by dark emitting, bulky fluorescent proteins and uncontrollable expression levels.

6 Here, we demonstrate the labeling of endogenous proteins using nanobodies with site-specifically

7 engineered bright organic fluorophores, named fluorobodies. Their fast and fine-tuned intracellular

8 transfer by microfluidic cell squeezing allowed for low background, low toxicity, and high-

9 throughput. Multiplexed imaging of distinct cellular structures was facilitated by specific protein

10 targeting, culminating in live-cell super-resolution imaging of protein networks. The high-

11 throughput delivery of engineered nanobodies will open new avenues in visualizing native cellular

12 structures with unprecedented accuracy in cell-based screens. 
1

2 The localization of endogenous proteins in native settings is fundamental for quantitative

\section{Introduction}

spatiotemporal understanding of cellular homeostasis and dysfunction. Here, live-cell imaging is highly desired to visualize timely-balanced protein networks, avoiding contingent artifacts by fixation or permeabilization procedures. ${ }^{1}$ In this regard, genetic fusion of fluorescent proteins (FPs) enabled the visualization of proteins of interest (POIs). ${ }^{2}$ However, the fusion to FPs or other bulky enzyme-based tags (e.g. $\mathrm{SNAP}_{\mathrm{f}}$ tag) ${ }^{3}$ can provoke protein misassembly, mistargeting, and overexpression artifacts. ${ }^{4}$ Alternatively, antibodies and recombinant binders are prominent tools to trace proteins. ${ }^{5-8}$ Yet, targeting with conventional antibodies is limited to fixed cells since their disulfide bonds are reduced in the cytosol. To overcome this downside, antibody fragments have been developed for protein targeting. ${ }^{9,10}$ For in-cell labeling of endogenous proteins, the binder must fulfill several demands: high affinity and specificity to its target, long-term stability in the reducing milieu of the cytosol, and small size avoiding interference with the POl's function. In this light, the variable domains of heavy-chain only antibodies $\left(\mathrm{V}_{\mathrm{H}} \mathrm{Hs}\right)$ of camelids, named nanobodies, are prime candidates. ${ }^{11,12}$ They bind with nanomolar or even picomolar affinities to their targets, are very small in size $(\sim 13 \mathrm{kDa})$, exhibit great solubility, and can be functionally produced in the reducing cytosolic milieu of mammalian cells. Nanobodies fused to fluorescent proteins (chromobodies) have been used to trace various POls. ${ }^{12-18}$ Recently, a chromobody has been developed for actin labeling in the cytosol and nucleus of mammalian cells, ${ }^{19}$ surpassing common techniques such as LifeAct by minimizing interference with actin dynamics. ${ }^{20}$ Nonetheless, visualization of intracellular proteins is still impeded because fused FPs triple the size of the nanobody and FPs entail suboptimal photophysical properties for super-resolution microscopy. An additional drawback of chromobodies is that their expression level cannot be precisely controlled, evoking high background and deteriorated signal-to-background staining, which in turn makes the observation of low abundant proteins and in particular super-resolution microscopy very difficult or impossible. As recently demonstrated, nanobodies directed against FPs were 
1 equipped with quantum dots or organic dyes to trace kinesin by single-particle tracking. ${ }^{21,22}$

2 However, both studies were limited by the expression of a genetically modified POI.

3 So far, methods visualizing endogenous proteins in live unmodified cells have not been

4 accomplished. To overcome this limitation, we advanced nanobodies with bright, high-

5 performance fluorescent probes, named fluorobodies. Combined with high-throughput cellular

6 delivery by a vector-free microfluidic device, ${ }^{23,24}$ endogenous proteins were visualized by multi-

7 color imaging at excellent signal-to-background ratios. Ultimately, the endogenous nuclear lamina

8 was resolved at nanometer precision by live-cell super-resolution microscopy.

\section{Results}

\section{Specific protein labeling via fluorobodies}

12 As a first showcase, we modified the established $\alpha$-GFP nanobody ${ }^{25,26}$ by strategic positioning of 13 small organic fluorescent probes. We engineered nanobodies with a single free cysteine either at 14 the $\mathrm{N}$ or $\mathrm{C}$ terminus, or position 9 , respectively. After expression and affinity purification, the 15 nanobodies were labeled via maleimide coupling with high-quantum yield fluorophores of, e.g. sulfo-Cyanine 3 (sCy3), sCy5, or ATTO655 (Figure 1a). To suppress transient exposure of inner

17 scaffold cysteines by thermal breathing, covalent dye attachment was performed at $4{ }^{\circ} \mathrm{C} .^{27}$

18 Specific conjugation of the fluorophore was confirmed by SDS-PAGE in-gel fluorescence and

19 fluorescence-detection size-exclusion chromatography, with molar labeling ratios of $\sim 0.9$ for

$20 \alpha-G F P^{s C y 3}$ and $\sim 0.8$ for $\alpha-G F P^{s C y 5}$ (Figure 1 b and Figure 1 - Figure Supplement 1).

21 As an initial screen, we evaluated specific binding of the a-GFP fluorobodies (Fbs) in fixed cells

22 prior to live-cell applications. Different intracellular assemblies were selected as targets, e.g. the

23 transporter associated with antigen processing TAP (TAP1-mVenus) in the ER membrane,

24 histone 2B (H2B-EGFP) in the nucleus, and lamin A (mEGFP-Lamin A) as a component of the 
1 nuclear envelope. The respective POls were expressed in human HeLa Kyoto cells, labeled with

2 the $\alpha$-GFP Fbs (50-200 nM) and analyzed by confocal laser scanning microscopy (CLSM). The

3 Fbs retained high target specificity with negligible background staining (Figure 1c,d and Figure 1

4 - Figure Supplement 2). More important, an excellent co-localization was observed as depicted

5 by Pearson's coefficients ranging from 0.948 to 0.986 , independently of the attached synthetic

6 dyes (sCy3 and sCy5; Figure 1c,d and Figure 1 - Figure Supplement 2). The Fb intensity

7 distribution highly correlated with the POI expression level, reflecting specific and stoichiometric

8 binding (Figure 1e and Figure 1 - Figure Supplement 2 and 3).

9 To avoid limitations typically entailed by overexpression of fusion proteins, we traced the endogenous nuclear envelope by an engineered $\alpha$-Lamin Fb. As basic constituents of the nuclei,

11 lamins are assembled in a filamentous meshwork, providing structural stability. Importantly, only

12 after nanobody production in an E. coli strain, engineered to promote disulfide bond formation in

13 the cytosol, and application of an optimized coupling procedure, specific labeling of the

14 endogenous nuclear lamina was facilitated with a-Lamin Fbs equipped with sCy5 or ATTO655 at

15 labeling ratios of 0.8 to 1.0 , respectively. Noteworthy, different procedures led to successful dye

16 conjugation, but were accompanied with loss in specificity (Figure 1 - Figure Supplement 4).

17 Similar results were obtained if lysine residues on the nanobody surface were stochastically

18 modified via N-hydroxysuccinimide (NHS) Alexa647 (Figure 1 - Figure Supplement 4a).

19 Contrary, the a-GFP Fb always retained high target specificity in fixed cells, independent of all

20 described routes for production and labeling (Figure 1 - Figure Supplement 5). Since the highest

21 signal-to-background ratio was achieved after dye-conjugation to the $\mathrm{N}$-terminal cysteine of the

22 a-Lamin Fb (Figure 1f,g; Supplementary Video 1 and Figure 1 - Figure Supplement Fig. 4),

23 labeling at this position was employed for all further experiments. 


\section{Live-cell protein labeling at nanomolar fluorobody concentrations}

2 Once the specificity in fixed cells was proven, we aimed at live-cell protein labeling. For the fast

3 and efficient Fb transfer, we made use of high-throughput, microfluidic cell squeezing. Here,

4 transient pore formation of the plasma membrane is induced by forcing cells through micrometer

5 constrictions of microfluidic devices (Figure 2 - Supplement Figure 1). This approach has shown

6 to bypass endosomal uptake and offered precise control of the transduced cargo concentration

7 combined with high-throughput delivery $\left(1,000,000\right.$ cells/s) and minimal cytotoxicity $(<10 \%){ }^{23,24}$

8 Initially, $\alpha$-GFP Fbs were delivered into HeLa Kyoto cells expressing the three selected FP-tagged

9 proteins and the intracellular Fb distribution was analyzed 1 to $3 \mathrm{~h}$ after cell transfer. In all cases, the fluorescence of the $\alpha$-GFP Fb showed a high degree of co-localization with FP-tagged H2B,

11 TAP1, or lamin A, reflected by Pearson's coefficients ranging from 0.864 to 0.911 (Figure 2a and Figure 2 - Supplement Figure 2a). Exemplary, an excellent correlation between the fluorescence intensity profiles of TAP1-mVenus and the a-GFP ${ }^{s C y 3} \mathrm{Fb}$ was observed (Figure 2b,c; Pearson's coefficient of 0.926 ). Notably, all transduced cells displayed a very low background staining and the intensity of the $\mathrm{Fb}$ reflected the variability in the POI expression level. We observed an increased $\mathrm{Fb}$ binding in cells with high levels of FP-tagged POIs, whereas cells expressing low amounts of the POls displayed weaker fluorescence intensities (Figure 2 - Supplement

Figure 3). One explanation for this correlation is found in the release of unbound binders during membrane resealing as well as in the nanomolar concentration of Fbs in the squeezing buffer, both synergistically contributing to a high signal-to-background staining (Figure $2 \mathrm{~d}$ ). ${ }^{22}$ Indeed, untransfected cells indicated a very low level of uniformly intracellular distributed Fbs (Figure 2 Supplement Figure 4). This is in line with the localization of the a-GFP-mCherry chromobody or mCherry expressed in HeLa Kyoto cells (Figure 2 - Supplement Figure 5a,b). In contrast, expression of $\alpha$-GFP-mCherry chromobodies together with FP-tagged POls often resulted in overabundance of the binder due to mismatched expression levels (Figure 2 - Supplement Figure 5c-e). Here, a high signal-to-background labeling is impaired in cells with high expression 
1 levels of chromobodies, while very low levels entail high sensitivity to photobleaching. These

2 drawbacks were successfully abolished by microfluidic $\mathrm{Fb}$ transfer. To test the long-term stability

3 and persistence of $\mathrm{Fb}$ labeling, cells were imaged $20 \mathrm{~h}$ after transduction. Remarkably, the POls

$4 \quad$ were still decorated by $\alpha$-GFP Fbs, highlighting their long-term specificity and in-cell robustness

5 (Figure 2 - Supplement Figure 2). Over time, however, cytosolic punctae and a higher

6 background signal were detected, indicating incipient Fb degradation. Collectively, the adaptive,

7 fine-tuned intracellular delivery of Fbs by cell squeezing promoted low-background labeling of

8 genetically tagged proteins.

High signal-to-background tracing of endogenous proteins in live mammalian cells

11 We next focused on live-cell labeling of endogenous protein networks. With regard to their 12 extenuated abundance, the utilization of high-performance fluorescent or fluorogenic probes is 13 essential to enhance sensitivity. We selected two intermediate filaments as targets. Specifically, 14 we used the engineered $\mathrm{a}$-Lamin $\mathrm{Fb}$ to visualize the native lamin meshwork of the nuclear 15 envelope and $\alpha$-Vimentin ${ }^{\text {ATTO488 }}$ for tracing vimentin as a cytoskeletal component, crucial for organelle positioning. Target specificity of both Fbs was verified beforehand in fixed cells

17 (Figure 1f,g and Figure 3 - Supplement Figure 1). In live HeLa Kyoto cells expressing a-Lamin-EGFP chromobody, decoration of the nuclear lamina was accompanied with high background staining and cytosolic punctae, presumably due to saturation of the targeting machinery (Figure 3 - Supplement Figure 2). Contrarily, specific decoration of endogenous

21 lamin with a concomitant negligible background was visualized $3 \mathrm{~h}$ after cell squeezing utilizing

22 the $\alpha$-Lamin ${ }^{\mathrm{ATTO}}{ }^{255} \mathrm{Fb}$ (Figure 3a). To the best of our knowledge this constitutes the first labeling 23 of an endogenous protein inside living cells by Fbs. Notably, even $20 \mathrm{~h}$ after cell squeezing, 24 labeling of the nuclear lamina was still persistent. Nevertheless, higher background, mainly as 25 cytosolic punctae, was observed (Figure 3 - Supplement Figure 3), in line with results obtained 
1 for the a-GFP Fb (Figure 2 - Supplement Figure 2b). Of note, specific targeting was unaffected

2 by the utilized fluorophore (ATTO655 or sCy5; Figure 3a and Figure 3 - Supplement Figure 3).

3 To further broaden the range of applications, $\alpha$-Vimentin ${ }^{\text {ATTO488 }}$ was delivered into cells. The well-

4 defined filamentous structure of endogenous vimentin was visualized with a high signal-to-

5 background ratio $3 \mathrm{~h}$ as well $20 \mathrm{~h}$ after cell squeezing (Figure 3b,c and Figure $\mathbf{3}$ - Supplement

6 Figure 4).

9 Encouraged by these results, we performed multi-color live-cell labeling by combining both 10 binders. 3D mapping of cell topology via multiplexed imaging revealed simultaneous and specific 11 decoration of the nuclear lamina (Figure $\mathbf{3 d}$, red) and vimentin filaments (Figure $\mathbf{3} \mathbf{d}$, green) at 12 endogenous level, hardly realized by multiple knock-ins utilizing the CRISPR/Cas9 technology.

13 The spatial distinct distribution allowed discriminating between both structures by live-cell 14 immunofluorescence imaging. In combination with their precisely adjusted delivery by cell 15 squeezing, the small Fbs, site-specifically labeled with minimal and bright fluorescent dyes $16(\leq 1 \mathrm{~nm})$ provides an excellent showcase for live-cell super-resolution microscopy. Consequently,

$17 \alpha$-Lamin ${ }^{\text {ATTO655 }} \mathrm{Fb}$ transduced cells were subjected to direct stochastic optical reconstruction microscopy (dSTORM). ${ }^{28}$ We obtained images of the endogenous nuclear lamina in living cells with an average resolution of $\sim 55 \mathrm{~nm}$ (localization precision of $23.4 \pm 8.3 \mathrm{~nm}$; Figure $3 \mathrm{e}$ ) and significantly increased contrast compared to wide-field imaging (Figure 3f). Strikingly, dSTORM analysis of $\alpha$-Lamin ${ }^{\mathrm{ATTO}}{ }^{\mathrm{S} 55} \mathrm{Fb}$ in fixed cells yielded virtually identical average localization precision, 
1

2

3

4

\section{Discussion}

In general, the much lower level of endogenous proteins makes their specific and low-background tracing very challenging. To circumvent this constrain, we established a method for live-cell visualization of endogenous proteins at nanometer resolution and with minimal disturbance and high reproducibility ( $n \geq 3$ for every POI). This robust and generic technique is versatile in the choice of the nanobody and bright organic fluorophore and allows for low-background tracing of high as well as low abundant native protein networks in living cells. In case of our selected target structures, Fb decoration was persistent for several hours after Fb transfer. However, increased background after $20 \mathrm{~h}$ suggests that long-term protein tracing can be affected. This obstacle is experimentally manageable, depending on the turnover rate of the POI as well as the rate of cell division. Alternative approaches to tag endogenous proteins such as the generation of stable cell lines utilizing e.g. CRISPR/Cas9 for low chromobody levels are highly promising, yet very laborintensive and hardly realizable for multiple knock-ins.

Collectively, our devised strategy strikes a new path for the live-cell visualization of endogenous targets by high quantum yield fluorobodies to study their precise intracellular localization and trafficking. The simplicity and robustness of the methodology can be extended to deliver multiple probes simultaneously for multiplexed imaging and has tremendous potential for other types of microscopy (e.g. electron microscopy). Even the $\mathrm{Fb}$ targeting of endogenous proteins in embryonic stem cells or patient-derived primary immune cells is technically feasible via cell squeezing. Moreover, our method provides access of exogenously derived high-affinity binding scaffolds (e.g. fibronectin-based binding proteins or designed ankyrin repeat proteins) for 3D and multi-color super-resolution imaging in native cells and opens avenues to label cellular ultrastructures at endogenous level for correlated light and electron microscopy (CLEM) or lattice light-sheet microscopy. Overall, the readily and feasible expansion of the methodology will pioneer the non-genetically encoded visualization of cellular networks and subcellular structures and 
hence provide complementary and unique information at unprecedented precision. Thus, fluorobody delivery by cell squeezing for in-cell tracing will likely find applications supporting routine imaging of multiple native cellular assemblies with advanced imaging techniques.

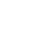

Acknowledgement

The German Research Foundation (Cluster of Excellence EXC 115 - Macromolecular Complexes to R.W., M.H, and R.T.; SPP 1623, RTG 1986 to R.T. and CRC 807 to M.H. and R.T.) and the

Volkswagen Foundation (A117158 to M.H., R.W., and R.T.) supported this work. We thank Dr. Eric Geertsma (Goethe University Frankfurt, Germany) for providing the sequence coding for the a-GFP nanobody and helpful discussions. We thank Dr. Heinrich Leonhardt (LMU Munich, Germany) for generously providing the plasmid coding for $\alpha$-Lamin-EGFP.

\section{Authors Contribution}

A.K. performed the nanobody labeling and cell squeezing experiments. S.H., A.K., and F.T. carried out the protein expression and purification. A.R. and M.H. performed the dSTORM imaging and analysis. A.K., R.W., and R.T. wrote the manuscript and analyzed the data. R.W. and R.T. conceived the ideas and directed the work.

Competing Financial Interests The authors declare no competing financial interests.

\section{References}

1 Schnell, U., Dijk, F., Sjollema, K.A. \& Giepmans, B.N. Immunolabeling artifacts and the need for livecell imaging. Nat Methods 9, 152-158 (2012).

2 Cubitt, A.B. et al. Understanding, improving and using green fluorescent proteins. Trends Biochem Sci 20, 448-455 (1995).

3 Gautier, A. et al. An engineered protein tag for multiprotein labeling in living cells. Chem Biol 15, 128-136 (2008).

4 El-Husseini, A.E., Schnell, E., Chetkovich, D.M., Nicoll, R.A. \& Bredt, D.S. PSD-95 involvement in maturation of excitatory synapses. Science 290, 1364-1368 (2000). 

aCC-BY 4.0 International license.

5 Brauchle, M. et al. Protein interference applications in cellular and developmental biology using DARPins that recognize GFP and mCherry. Biol Open 3, 1252-1261 (2014).

6 Karatan, E. et al. Molecular recognition properties of FN3 monobodies that bind the Src SH3 domain. Chem Biol 11, 835-844 (2004).

7 Nygren, P.A. Alternative binding proteins: affibody binding proteins developed from a small threehelix bundle scaffold. FEBS J 275, 2668-2676 (2008).

8 Skerra, A. Engineered protein scaffolds for molecular recognition. J Mol Recognit 13, 167-187 (2000).

9 Bird, R.E. et al. Single-chain antigen-binding proteins. Science 242, 423-426 (1988).

10 Huston, J.S. et al. Protein engineering of antibody binding sites: recovery of specific activity in an anti-digoxin single-chain Fv analogue produced in Escherichia coli. Proc Natl Acad Sci U S A 85, 58795883 (1988).

11 Muyldermans, S. Single domain camel antibodies: current status. J Biotechnol 74, 277-302 (2001).

12 Helma, J., Cardoso, M.C., Muyldermans, S. \& Leonhardt, H. Nanobodies and recombinant binders in cell biology. J Cell Biol 209, 633-644 (2015).

13 Caussinus, E., Kanca, O. \& Affolter, M. Fluorescent fusion protein knockout mediated by anti-GFP nanobody. Nat Struct Mol Biol 19, 117-121 (2011).

14 Maier, J., Traenkle, B. \& Rothbauer, U. Real-time analysis of epithelial-mesenchymal transition using fluorescent single-domain antibodies. Sci Rep 5, 13402 (2015).

15 Zolghadr, K., Gregor, J., Leonhardt, H. \& Rothbauer, U. Case study on live cell apoptosis-assay using lamin-chromobody cell-lines for high-content analysis. Methods Mol Biol 911, 569-575 (2012).

16 Rothbauer, U. et al. Targeting and tracing antigens in live cells with fluorescent nanobodies. Nat Methods 3, 887-889 (2006).

17 Schornack, S. et al. Protein mislocalization in plant cells using a GFP-binding chromobody. Plant J 60, 744-754 (2009).

18 Tang, J.C. et al. Detection and manipulation of live antigen-expressing cells using conditionally stable nanobodies. elife 5 (2016).

19 Plessner, M., Melak, M., Chinchilla, P., Baarlink, C. \& Grosse, R. Nuclear F-actin formation and reorganization upon cell spreading. J Biol Chem 290, 11209-11216 (2015).

20 Melak, M., Plessner, M. \& Grosse, R. Actin visualization at a glance. J Cell Sci 130, 525-530 (2017).

21 Katrukha, E.A. et al. Probing cytoskeletal modulation of passive and active intracellular dynamics using nanobody-functionalized quantum dots. Nat Commun 8, 14772 (2017).

22 Teng, K.W. et al. Labeling proteins inside living cells using external fluorophores for microscopy. elife 5 (2016).

23 Kollmannsperger, A. et al. Live-cell protein labelling with nanometre precision by cell squeezing. Nat Commun 7, 10372 (2016).

24 Sharei, A. et al. A vector-free microfluidic platform for intracellular delivery. Proc Natl Acad Sci U S A 110, 2082-2087 (2013).

25 Ries, J., Kaplan, C., Platonova, E., Eghlidi, H. \& Ewers, H. A simple, versatile method for GFP-based super-resolution microscopy via nanobodies. Nat Methods 9, 582-584 (2012).

26 Kirchhofer, A. et al. Modulation of protein properties in living cells using nanobodies. Nat Struct Mol Biol 17, 133-138 (2010).

27 Pleiner, T. et al. Nanobodies: site-specific labeling for super-resolution imaging, rapid epitopemapping and native protein complex isolation. eLife 4, e11349 (2015).

28 Heilemann, M. et al. Subdiffraction-resolution fluorescence imaging with conventional fluorescent probes. Angew Chem Int Ed Engl 47, 6172-6176 (2008). 


\section{Methods}

\section{Plasmid construction}

4 For expression in E. coli, the a-GFP nanobody, ${ }^{26}$ equipped with a C-terminal His ${ }_{6}$-tag for

5 purification via metal ion affinity chromatography, was inserted into the pET21a(+) plasmid. For

6 site-specific labeling, a free single cysteine was introduced C-terminally to the His ${ }_{6}$-tag. The $\alpha$-GFP

7 nanobody was PCR amplified by the following primers: fw 5'-GCGCGCAAGCTTAAGG

8 AGATATACATATGCAGGTTCAGCTGGTTGAAAGCGGTGGTGCAC-3' (Hind III restriction site

9 underlined, RBS italic, start codon bold); rev 5'-GCGCCTCGAGGTCAGCAGTGGTGATGG TGATGATGGCTGCTAACGGTAACCTGGGTGCCC-3' (Xhol restriction site underlined, stop codon bold, cysteine bold and underlined, His $_{6}$-tag italic). For expression in mammalian cells, a-GFP as well as mCherry were PCR amplified and introduced into the pcDNA3.1(+) plasmid, resulting in a plasmid coding for $\alpha$-GFP ${ }^{\text {mCherry }}$. fw 5'-GCGCGCGCTAGCACCATGCAGGTTCA GCTGGTTGAAAGCGG-3' (Nhel restriction site underlined, start codon bold) and rev 5'-GCGCGCGGTACCGCTGCTAACGGTAACCTGGGTGCCC-3' (Acc65I restriction site underlined) for the a-GFP nanobody and fw 5'-CGCGCGGTACCATGGTGAGCAAGGGCGAG GAGCTGTTC-3' (Acc65I restriction site underlined, start codon bold) and rev 5'-GCGCGCCTCGAGTTACTTGTACAGCTCGTCCATGCCGAGAG-3' (Xhol restriction site underlined, stop codon bold) for mCherry. The $\alpha$-Lamin nanobody was also inserted into the pET21a(+) plasmid and equipped with a $\mathrm{His}_{6}$-tag for purification. Using a-Lamin-EGFP as PCR

21 template, $\alpha$-Lamin was amplified, introducing a C-terminal cysteine ( $\alpha$-Lamin ${ }^{\text {His6-Cys }}$ ), a N-terminal cysteine $\left({ }^{\text {Cys }} \alpha\right.$-Lamin $\left.{ }^{\text {His6 }}\right)$, or replacing serine 9 by a cysteine $\left(\alpha\right.$-Lamin $\left.{ }^{\text {S9C-His6 }}\right)$. $\alpha$-Lamin ${ }^{\text {His6-Cys. }}$ fw 5'-GCGCAAGCTTAAGGAGATATACATATGGCTCAGGTACAGCTGCAGGAGTCTGGAGGA

24 GG-3' (HindIII restriction site underlined, start codon bold, RBS italic); rev 5'-GCGCCTCGAG 25 TtAACAGTGGTGATGGTGATGATGCGATATCGAATTCCTTGAGGAGACGGTGACCTGGG-3’ 26 (Xhol restriction site underlined, stop codon bold, cysteine bold and underlined, His 6 -tag italic). 
1 To attach the PelB leader sequence and the cysteine, ${ }^{\text {Cys }} \alpha$-Lamin ${ }^{\text {His6 }}$ were amplified in consecutive extending PCRs, each of them being the template for the subsequent amplification. The rev primer 5'-GCGCGCCTCGAGTTAGTGGTGATGGTGATGATGGGTGGCGACCGGCCG-3' restriction site underlined, stop codon bold, $\mathrm{His}_{6}$-tag italic) was thereby applied in combination with the following fw primers. ${ }^{\text {Cys }} \alpha$-Lamin ${ }^{\text {His6. }}$ fw1 $5^{\prime}$-GGATTGTTATTACTCGCGGC CCAGCCGGCCTGTGCTCAGGTACAGCTGCAGGAGTCTGGAGGAGG-3' (cysteine bold, part of pelB leader sequence italic), fw2 5'-GCGCGCTAGCATGAAATACCTATTGCCTACGGCAG CCGCTGGATTGTTATTACTCGCGGCCCAGCCGGCC-3' (Nhel restriction site underlined, start

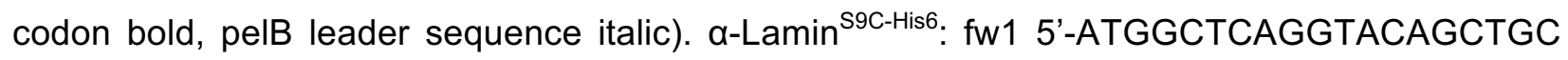
AGGAGTGTGGAGGAGGCTTGGTGCAGGCAGGGGGGTCTCTG-3'， fw2 5'-GGATTGTTAT TACTCGCGGCCCAGCCGGCCATG GCTCAGGTACAGCTGCAGGAGTGTGGA-3' (cysteine bold, part of pelB leader sequence italic), fw3 5'-GCGCGCTAGCATGAAATACCTATT GCCTACGGCAGCCGCTGGATTGTTATTACTCGCGGCCCAGCCGGCC-3' (Nhel restriction site underlined, start codon bold, pelB leader sequence italic). The plasmid coding for H2B-EGFP was obtained from Addgene (plasmid 11680). The generation of plasmids coding for core TAP1 (TAP1mVenus) and mEGFP-Lamin A was described previously. ${ }^{23,29}$

\section{Nanobody production}

The nanobodies were produced in E. coli BL21 (DE3, Life technologies) or E. coli SHuffle T7 Express (New England Biolabs), whereas the latter contains a chromosomal copy of a disulfide isomerase, engineered to produce proteins with disulfide bonds in the cytosol. Both $E$. coli strains were transformed with the vectors, coding for $\alpha$-GFP and $\alpha$-Lamin nanobodies with engineered cysteines. A single colony was inoculated in LB medium containing $100 \mu \mathrm{g} / \mathrm{ml}$ ampicillin and $2 \%(w / v)$ glucose. After overnight incubation $\left(37^{\circ} \mathrm{C}\right.$ for $E$. coli BL21 (DE3) and $30{ }^{\circ} \mathrm{C}$ for E. coli SHuffle T7 Express, $180 \mathrm{rpm}$ ), 1 I Lysogeny Broth (LB) medium with $100 \mu \mathrm{g} / \mathrm{ml}$ ampicillin was inoculated and protein expression was induced at $A_{600}$ of $0.7-0.8$ by $1 \mathrm{mM}$ isopropyl- $\beta$ - 
1 thiogalactopyranoside (IPTG, Sigma-Aldrich) and conducted for 5-20 h (30 $\left.{ }^{\circ} \mathrm{C}, 180 \mathrm{rpm}\right)$. After

2 centrifugation $\left(20 \mathrm{~min}, 4^{\circ} \mathrm{C}, 5,000 \mathrm{xg}\right.$ ), the cell pellets were directly used for protein purification or

3 stored at $-20{ }^{\circ} \mathrm{C}$.

4

5 Protein purification

6 Two different protocols were used for purification. For $\alpha-$ GFP $^{\text {His6-Cys }}$ and $\alpha$-Lamin ${ }^{\text {His6-Cys }}$ expressed

7 in E. coli BL21 (DE3), cell pellets were resuspended in lysis buffer $(20 \mathrm{mM}$ Tris $\mathrm{pH} 8.0$,

$8100 \mathrm{mM} \mathrm{NaCl}, 1 \mathrm{mM}$ phenylmethylsulfonyl fluoride (PMSF), $0.5 \mathrm{mM} \beta$-mercaptoethanol ( $\beta-\mathrm{ME})$ ),

9 followed by sonication for cell lysis. Cell debris were pelleted by ultracentrifugation (100,000x $\mathrm{g}$,

$1030 \mathrm{~min}, 4^{\circ} \mathrm{C}$ ). For metal affinity chromatography, the supernatant was incubated with $3 \mathrm{ml} \mathrm{Ni-NTA}$

11 Sepharose beads (GE Healthcare) in the presence of imidazole (15 mM). Afterwards, the beads

12 were washed in purification buffer (50 mM Tris $\mathrm{pH} 8.0,300 \mathrm{mM} \mathrm{NaCl}, 50 \mathrm{mM}$ imidazole, $0.5 \mathrm{mM}$

$13 \beta-M E)$. His-tagged proteins were eluted by $200 \mathrm{mM}$ imidazole/purification buffer. Protein

14 concentration was determined at $A_{280}$. Buffer exchange to phosphate-buffered saline (PBS)

15 containing $0.5 \mathrm{mM} \beta-\mathrm{ME}$ by Zeba spin desalting columns (7K) was followed by concentration via

16 Amicon Ultra Filters (3 K). $\beta$-ME was directly removed before labeling the nanobodies using Zeba

17 spin desalting columns (7K). Alternatively, nanobodies expressed in E. coli T7 Express were

18 purified as described above, but at $\mathrm{pH} 7.5$ and without $\beta-\mathrm{ME}$.

\section{Protein modification}

21 Fluorescent dyes were either attached to the engineered cysteines or to lysines (only for

$22 \alpha$-Lamin $\left.{ }^{\text {His6-Cys }}\right)$. The engineered cysteines of nanobodies stored in PBS without $\beta$-ME were

23 reduced by incubation with $15 \mathrm{mM}$ TCEP for $10 \mathrm{~min}$ on ice and subsequent buffer exchange by

24 Zeba spin desalting columns (7K). Cysteine labeling was conducted following two different

25 protocols. On the one hand, nanobodies were incubated with sulfo-Cy5 (sCy5) or sulfo-Cy3 (sCy3) 
maleimide in PBS $(\mathrm{pH} 7.4)$ for $1.5 \mathrm{~h}$ at $4{ }^{\circ} \mathrm{C}$. Labeling was conducted at a protein concentration of approximately $1 \mathrm{mg} / \mathrm{ml}$ with 1.2 -fold molar excess of the dye. For the alternative labeling protocol,

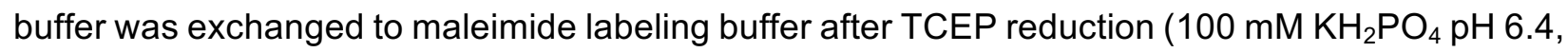
$150 \mathrm{mM} \mathrm{NaCl}, 1 \mathrm{mM}$ EDTA, $250 \mathrm{mM}$ sucrose). Afterwards, the fluorophore was added in a 1.2fold molar excess, immediately followed by neutralization to $\mathrm{pH} 7.5$ using $\mathrm{K}_{2} \mathrm{HPO}_{4}$ and $1.5 \mathrm{~h}$ incubation at $4{ }^{\circ} \mathrm{C}$. For lysine labeling, nanobodies in PBS $(\mathrm{pH} 7.4)$ were mixed 1:20 with $0.1 \mathrm{mM}$ $\mathrm{NaHCO}_{3}(\mathrm{pH}$ 9.0) to achieve $\mathrm{pH} 8.3$ for labeling. Next, Alexa647 NHS ester was added with a 5-fold molar excess. Labeling was performed for $1 \mathrm{~h}$ at $20^{\circ} \mathrm{C}$ before buffer exchange and removal of excess dye with Zeba spin desalting columns (7K, Thermo Scientific). Fluorescently labeled nanobodies were analyzed by SDS-PAGE and in-gel fluorescence (Typhoon 9400 Imager; $\lambda_{\text {ex/em }}$ $630 / 670 \mathrm{~nm})$. Fbs were finally purified by size-exclusion chromatography in PBS using a KW404-4F column (Shodex; flow rate $0.3 \mathrm{ml} / \mathrm{min}$ ). All labeled nanobodies were stored in PBS at $4{ }^{\circ} \mathrm{C}$. The nanobody-to-fluorophore ratio was determined using absorption $\left(\mathrm{A}_{280}\right.$ for nanobodies, $\mathrm{A}_{550}$ for $\mathrm{sCy} 3, \mathrm{~A}_{663}$ for $\mathrm{ATTO655}, \mathrm{A}_{649}$ for $\left.\mathrm{sCy} 5\right)$ and their respective extinction coefficient $\varepsilon\left(0.22 \cdot 10^{5} \mathrm{M}^{-1} \cdot \mathrm{cm}^{-1}\right.$ for $\alpha$-Lamin $\mathrm{Nb}, 0.27 \cdot 10^{5} \mathrm{M}^{-1} \cdot \mathrm{cm}^{-1}$ for $\alpha$-GFP Nb, $1.36 \cdot 10^{5} \mathrm{M}^{-1} \cdot \mathrm{cm}^{-1}$ for sCy3, $1.25 \cdot 10^{5} \mathrm{M}^{-1} \cdot \mathrm{cm}^{-1}$ for ATTO655, and $2.50 \cdot 10^{5} \mathrm{M}^{-1} \cdot \mathrm{cm}^{-1}$ for sCy5).

\section{Cell culture and transfection}

HeLa Kyoto cell lines were cultivated in Dulbecco's Modified Eagle Medium (DMEM) with 4.5 g/l glucose (Gibco) containing 10\% (v/v) fetal calf serum (FCS, Gibco). Cells were regularly tested for Mycoplasma contamination. ${ }^{30}$ Cells were transiently transfected using Lipofectamine 2000

22 (Life technologies), following the manufacturer's instructions. For staining of fixed cells, $2 \cdot 10^{4}$ cells per well were seeded into 8-well on cover glass II slides (Sarstedt) and transfected at $~ 80 \%$ confluency. For cell squeezing, $8 \cdot 10^{5}$ cells were seeded into 6-well cell culture plates (Greiner) culture conditions until experiments were performed. 

10-20 min at $20{ }^{\circ} \mathrm{C}$ and permeabilized using $0.1 \%$ Triton X-100 (Roth)/PBS (10 min, $\left.20{ }^{\circ} \mathrm{C}\right)$. After blocking with $5 \%(\mathrm{w} / \mathrm{v})$ bovine serum albumin (BSA; Albumin Fraction $\mathrm{V}$, Roth) in PBS for $1 \mathrm{~h}$ at $20{ }^{\circ} \mathrm{C}$, cells were stained. Staining was performed with fluorobodies (diluted in $1 \%$ BSA/PBS) at concentrations ranging from 50 to $500 \mathrm{nM}$ (indicated in each case). Subsequently, cells were washed with PBS and optionally stained with $0.1 \mu \mathrm{g} / \mathrm{ml}$ 4',6-diamidino-2-phenylindole (DAPI, Sigma-Aldrich) in $1 \% \mathrm{BSA} / \mathrm{PBS}$ for $30 \mathrm{~min}$ to $1 \mathrm{~h}$ at $20^{\circ} \mathrm{C}$. After washing with $5 \% \mathrm{BSA} / \mathrm{PBS}(3 \mathrm{x})$, cells were post-fixed with $2 \%$ formaldehyde/PBS $\left(15 \mathrm{~min}, 20^{\circ} \mathrm{C}\right.$ ) and stored in PBS until imaging was performed.

\section{Confocal laser scanning microscopy}

Imaging was performed using the confocal laser scanning (LSM) microscope LSM880 (Zeiss) or

TCS SP5 microscope (Leica) with Plan-Apochromat 63x/1.4 Oil DIC objectives. To avoid crosstalk between different fluorophores, images were acquired sequentially. The following laser lines were used for excitation: $405 \mathrm{~nm}$ for DAPI; $488 \mathrm{~nm}$ for EGFP, mEGFP, and mVenus; $565 \mathrm{~nm}$ for sCy3; $633 \mathrm{~nm}$ for sCy5 and ATTO655. Live-cell imaging of endogenous proteins (except for dual-color imaging with ATTO488 and ATTO655) was conducted with the Airy scan detector of the LSM880 in the SR-mode. For 3D reconstructions of labeled protein networks, z-stack were recorded with the Airy scan detector (distance between stacks $200 \mathrm{~nm}$ ). For live-cell imaging, an incubation system was used to keep cells at $37^{\circ} \mathrm{C}$ and supply them with $5 \% \mathrm{CO}_{2}$ during imaging. Image ${ }^{31}$,

$22 \mathrm{Fiji}^{32}$, and Zen 2.3 black (Zeiss) were used for image analysis and to analyze Pearson's

23 coefficients from 8-11 individual cells per condition. The mean and standard deviation were calculated. 


\section{Fluorobody transfer by cell squeezing}

2 Squeezing was performed using a chip with constrictions of $7 \mu \mathrm{m}$ in diameter and $10 \mu \mathrm{m}$ in length

3 (CellSqueeze 10-(7)x1, SQZbiotech). In all microfluidic experiments, a cell density of $1.510^{6}$

$4 \mathrm{cells} / \mathrm{ml}$ in $10 \%(\mathrm{v} / \mathrm{v})$ FCS/PBS was squeezed through the chip at a pressure of 30 psi.

5 Transduction was conducted at $4{ }^{\circ} \mathrm{C}$ to block cargo uptake by endocytosis. ${ }^{23}$ During squeezing,

6 the following $\mathrm{Fb}$ concentrations in the surrounding buffer were used: 100-200 nM of $\alpha-\mathrm{GFP}$,

$7200-500 \mathrm{nM}$ of $\alpha$-Lamin, and $50 \mu \mathrm{g} / \mathrm{ml}$ of $\alpha$-Vimentin. After squeezing, cells were incubated for

$85 \mathrm{~min}$ at $4{ }^{\circ} \mathrm{C}$ to reseal the plasma membrane. Squeezed cells were washed with DMEM

9 containing 10\% FCS, seeded into 8-well on cover glass II slides (Sarstedt) or collagen-coated

10 dishes ( $\mu$-Slide 8 Well, Collagen IV coverslip, ibidi) in DMEM containing $10 \%$ FCS, and cultured

11 at $37{ }^{\circ} \mathrm{C}$ and $5 \% \mathrm{CO}_{2}$. Confocal imaging was performed $1 \mathrm{~h}$ and $20 \mathrm{~h}$ after squeezing. Squeezing

12 experiments for live-cell protein labeling highly reproducible in at least three independent

13 experiments for every POI.

\section{Super-resolution microscopy}

For super-resolution imaging of the $\alpha$-Lamin ${ }^{\mathrm{ATTO655}} \mathrm{Fb}$ in live cells, a custom-built microscope was emission was recorded using an EMCCD camera (IxonUltra, Andor) with frame-transfer mode, 1x pre-amplifier gain and EM gain set to 200. For every sample, 40,000 images were recorded at a

23 frame rate of $33 \mathrm{~Hz}$. Image reconstruction was performed with rapidSTORM ${ }^{33}$ using the smooth

24 by differences of averages option with a signal-to-noise ratio of 20 , fitting only PSFs with FWHM

25 ranging from $240-520 \mathrm{~nm}$ and $\mathrm{X}-\mathrm{Y}$-ratio of $0.7-1.3$. The average localization precision $\left(\sigma_{\text {loc }}\right)$ defined

26 as the nearest neighbor distance in adjacent frames ${ }^{34}$ was calculated to $23.4 \pm 8.3 \mathrm{~nm}$ for living 
and $23.2 \pm 4.6 \mathrm{~nm}$ for fixed cells (mean and standard deviation of three individually analyzed cells, respectively) using LAMA software. ${ }^{35}$ The average image used for visualization of nuclear intensity profiles in Figure $3 f$ was generated by averaging the fluorescence intensity over 100 frames and subsequent background subtraction with a rolling ball method (20 pixel radius) in ImageJ and Fiji.

\section{Data availability}

The datasets generated during and/or analyzed during the current study are available from the corresponding author on reasonable request.

\section{Supplementary References}

23 Kollmannsperger, A. et al. Live-cell protein labelling with nanometre precision by cell squeezing. Nat Commun 7, 10372 (2016).

26 Kirchhofer, A. et al. Modulation of protein properties in living cells using nanobodies. Nat Struct Mol Biol 17, 133-138 (2010).

9 Parcej, D., Guntrum, R., Schmidt, S., Hinz, A. \& Tampé, R. Multicolour fluorescence-detection sizeexclusion chromatography for structural genomics of membrane multiprotein complexes. PLoS One 8, e67112 (2013).

30 Uphoff, C.C. \& Drexler, H.G. Detection of mycoplasma contamination in cell cultures. Curr Protoc Mol Biol 106, 2824 21-28 2414 (2014).

31 Girish, V. \& Vijayalakshmi, A. Affordable image analysis using NIH Image/ImageJ. Indian J Cancer 41, 47 (2004).

32 Schindelin, J. et al. Fiji: an open-source platform for biological-image analysis. Nat Methods 9, 676682 (2012).

33 Wolter, S. et al. Real-time computation of subdiffraction-resolution fluorescence images. J Microsc 237, 12-22 (2010).

34 Endesfelder, U., Malkusch, S., Fricke, F. \& Heilemann, M. A simple method to estimate the average localization precision of a single-molecule localization microscopy experiment. Histochem Cell Biol 141, 629-638 (2014).

35 Malkusch, S. \& Heilemann, M. Extracting quantitative information from single-molecule superresolution imaging data with LAMA - LocAlization Microscopy Analyzer. Sci Rep 6, 34486 (2016). 

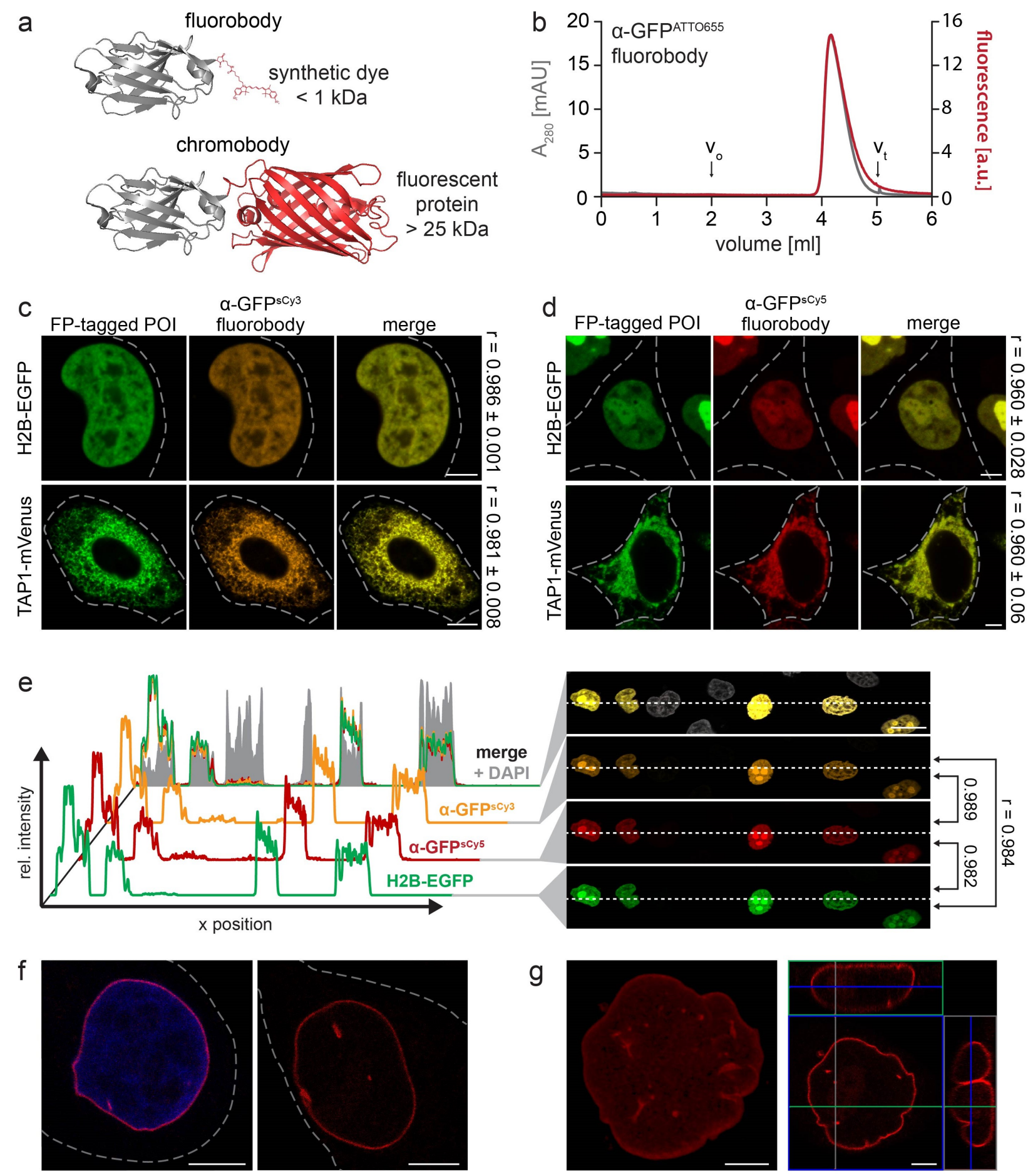

g
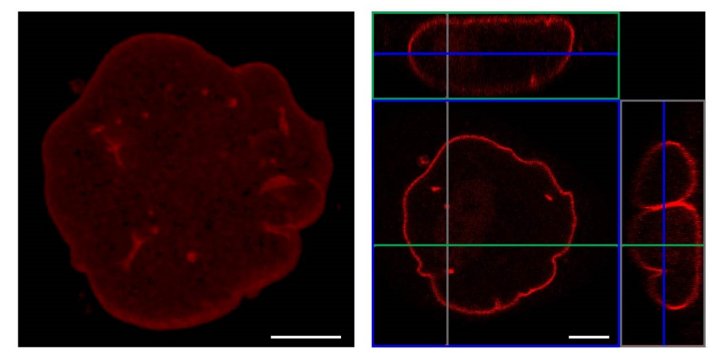

3 Figure 1 I Specific binding of site-specifically labeled fluorobodies. (a) Size comparison of 4 nanobodies labeled with a fluorescent protein (chromobody) or a synthetic dye (fluorobody). Bulky 5 fluorescent proteins (> $25 \mathrm{kDa})$ triple the size of the nanobody $(\sim 13 \mathrm{kDa})$. In comparison, the 
1 minimal size of fluorophores $(<1 \mathrm{kDa})$ is unlikely to disturb the nanobody and offer a higher 2 quantum yield as well as photostability. mCherry (PDB 2H5Q) is shown exemplarily. (b) Size3 exclusion chromatography (SEC) of the site-specifically labeled $\alpha-G F P^{A T T O 655} \mathrm{Fb}$. The grey and 4 red lines represent absorption at $280 \mathrm{~nm}\left(\mathrm{~A}_{280}\right)$ and ATTO655 fluorescence at $\lambda_{\text {ex/em }} 655 / 680 \mathrm{~nm}$.

5 Chromatogram shows specific modification of the nanobody and high purity. (c, d) Specific 6 labeling of FP-tagged proteins in chemically arrested cells. HeLa Kyoto cells expressing 7 H2B-EGFP or TAP1-mVenus (green) were fixed with 4\% formaldehyde and labeled with $100 \mathrm{nM}$ 8 of $\alpha-G F P^{s C y 3}$ (c, orange) or $\alpha-G^{-S^{s C y 5}}$ (d, red) Fb. Excellent co-localization (merge) between the 9 Fbs and both FP-tagged POls was detected. Pearson's coefficients ( $r$ ) were calculated from 9-11 10 individual cells (right). (e) Fixed HeLa Kyoto cells expressing H2B-EGFP were stained with $11 \alpha-\mathrm{GFP}^{\mathrm{sCy} 3}$ (orange), $\alpha-\mathrm{GFP}^{\mathrm{sCy} 5}$ (red, $100 \mathrm{nM}$ each), and DAPI (blue). Cross-sections of relative 12 fluorescence intensity profiles of both Fbs (along dashed line) highly correlated with the expression 13 level of H2B-EGFP. Low background fluorescence was detected in untransfected cells (only DAPI 14 positive), supported by the Pearson's coefficients ( $r$ ) between different channels (right). (f, g) After 15 fixation, the endogenous nuclear lamina of HeLa Kyoto cells was visualized by $100 \mathrm{nM}$ of $\alpha$-Lamin ${ }^{\text {ATTO655 }} \mathrm{Fb}$ (red). (g) The Fb specifically decorated the nuclear envelope (DNA optionally 17 visualized via DAPI staining, blue). (f) 3D reconstruction of a z-stack showed a dense labeling of 18 the nuclear lamina along with a high signal-to-background ratio (see Supplementary Video 1). 19 Images were taken by CLSM with the Airy Scan detector ( $f$ and $g$ ). Dashed lines indicate the cell border. Scale bars: $5 \mu \mathrm{m}(\mathbf{c}, \mathbf{d}, \mathbf{f}, \mathbf{g})$ and $20 \mu \mathrm{m}(\mathbf{e})$. 

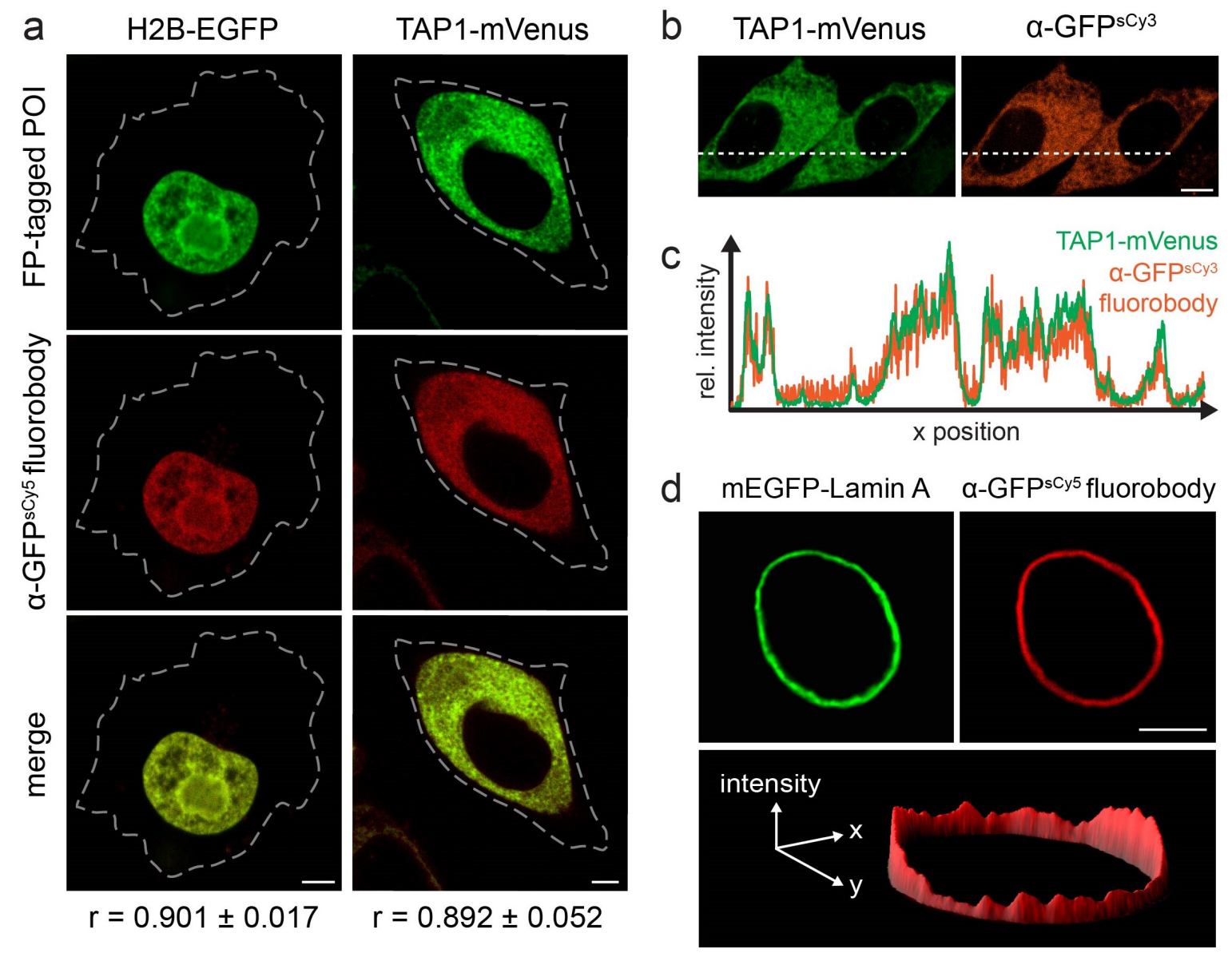

2 Figure 2 I Live-cell protein labeling with high-performance fluorobodies. (a) Protein labeling in 3 living cells at different subcellular localizations. The $\alpha-G F P^{s C y 5} \mathrm{Fb}(200 \mathrm{nM}$, red) was delivered via 4 cell squeezing into HeLa Kyoto cells, expressing H2B-EGFP or TAP1-mVenus (green). Excellent 5 co-localization (merge channel) of the Fbs with both FP-tagged POls was observed $3 \mathrm{~h}$ post 6 squeezing by CLSM. The Pearson's coefficients ( $r$, bottom) ranging from 0.864 to 0.905 were 7 calculated from 8-10 individual cells. Dashed lines indicate cell borders. (b) Live-cell visualization 8 of TAP1-mVenus (green) by $\alpha-\mathrm{GFP}^{\mathrm{sCy} 3} \mathrm{Fb}$ (orange) $20 \mathrm{~h}$ after squeezing. Cross-sections of 9 relative fluorescence intensity profiles of TAP1-mVenus and $\alpha-G^{-s^{s y} 3}$ (along dashed line) 10 showed specific and low-background labeling in live cells by the $\alpha$-GFP Fb (c). Pearson's 11 coefficient: 0.926. (d) 3D intensity plot of a representative HeLa Kyoto cell expressing 12 mEGFP-Lamin A (green) was stained by a-GFP ${ }^{s C y 5} \mathrm{Fb}$ (red) and showed a very high signal-to 13 background ratio. All images were taken by CLSM. Scale bar: $5 \mu \mathrm{m}$. 
a
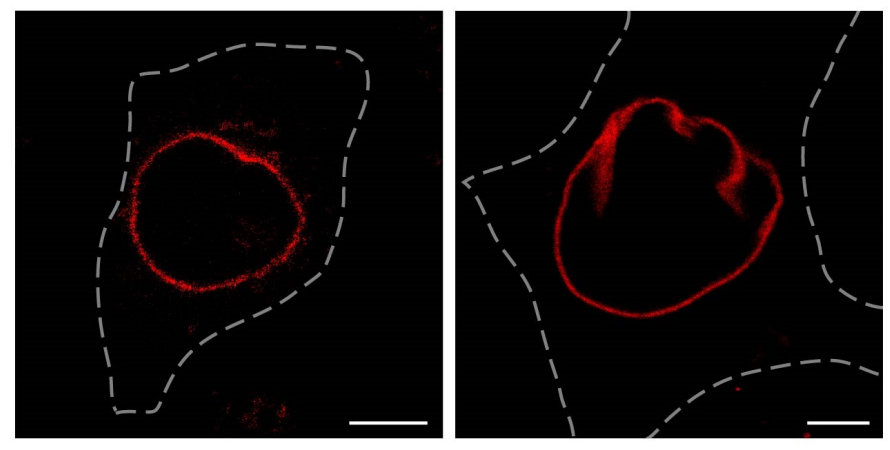

C
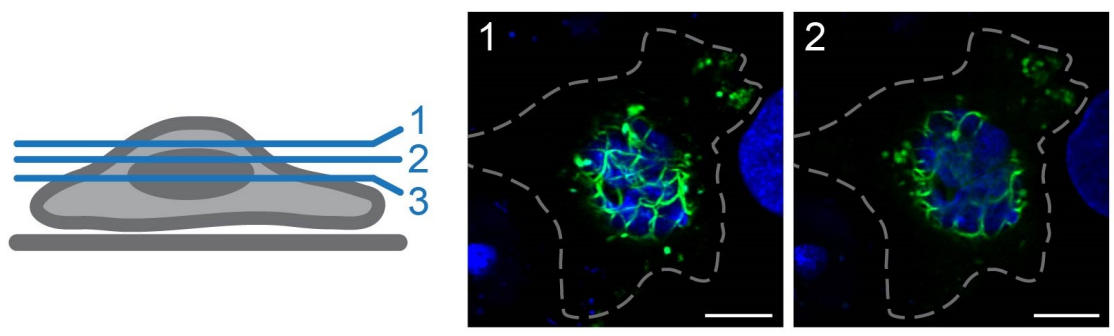

d
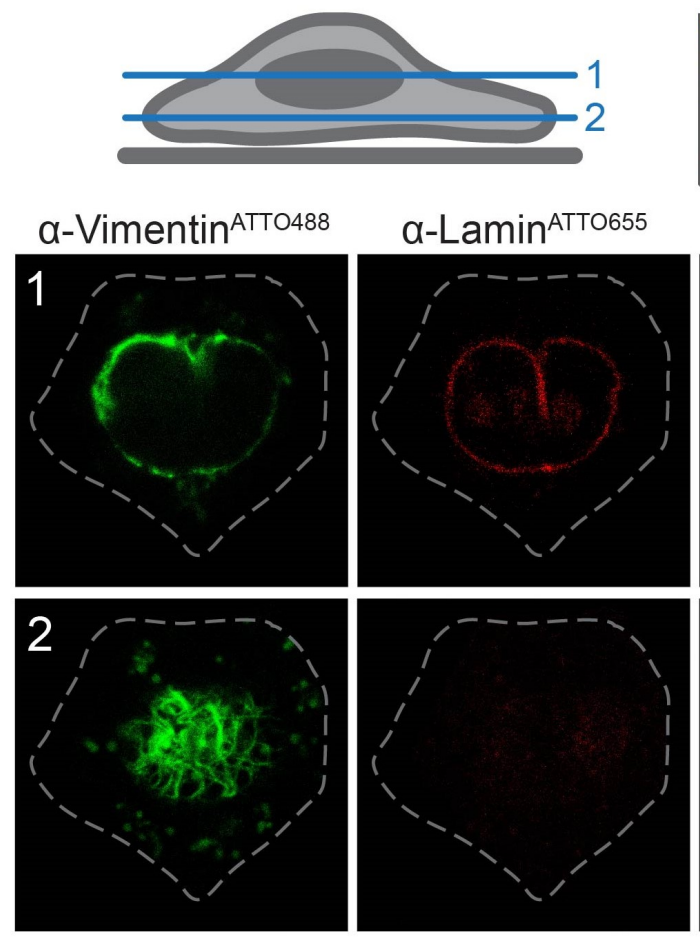
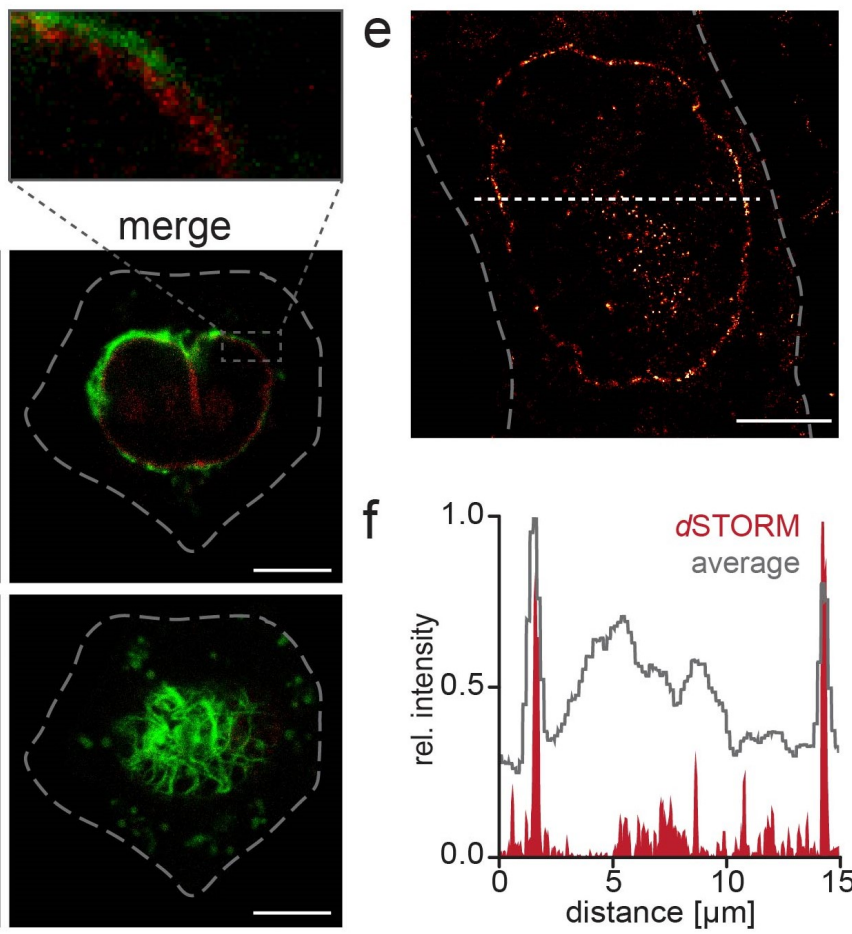
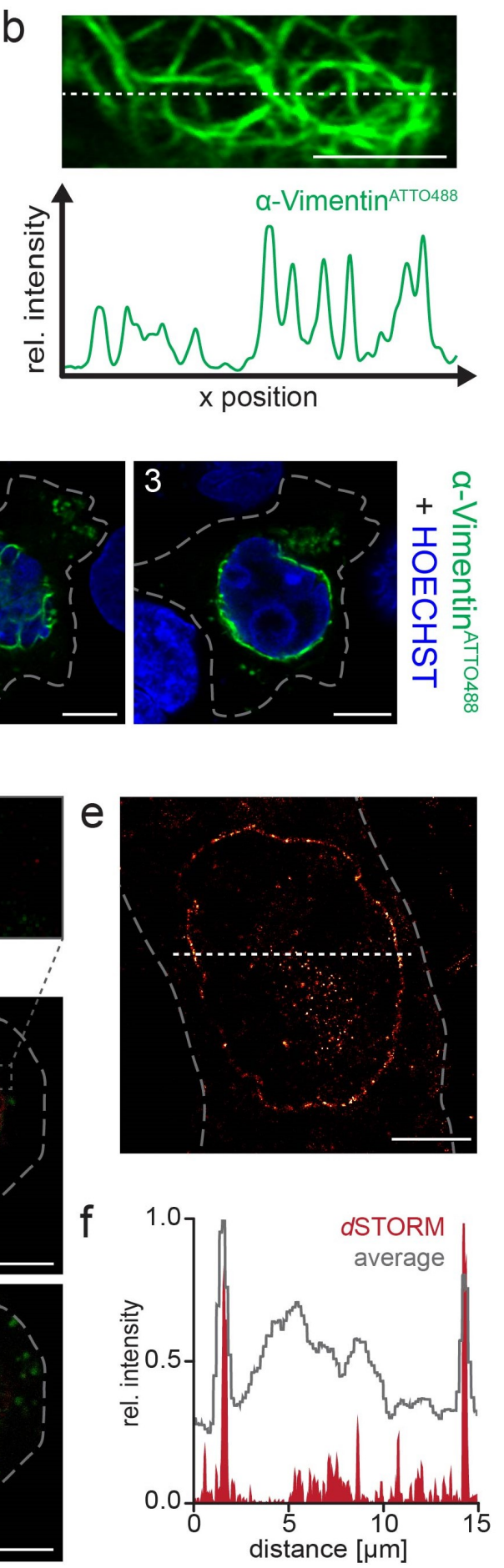

2 Figure 3 I Dual-color live-cell tracing of endogenous targets and live-cell super-resolution imaging 3 by fluorobodies. (a) The a-Lamin ${ }^{\text {ATTO655 }} \mathrm{Fb}(200 \mathrm{nM}$ ) was transferred into HeLa Kyoto cells via 4 squeezing. Confocal imaging after $3 \mathrm{~h}$ showed specific labeling of the native nuclear lamina in 5 living cells with high signal-to-background ratio. (b) HeLa Kyoto cells were squeezed with $50 \mu \mathrm{g} / \mathrm{ml}$ 
1 of $\alpha$-Vimentin ${ }^{\mathrm{ATTO} 488}$. The Fb decorated vimentin network was explicitly traced $3 \mathrm{~h}$ post squeezing 2 and visualized as a dense meshwork around the nucleus (HOECHST-positive, blue) in different

3 confocal z-planes (1-3; indicated in scheme, left). (c) Fluorescence intensity cross-section of a live

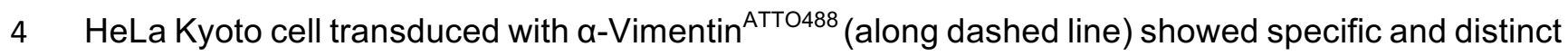

5 decoration of the endogenous vimentin meshwork with high signal-to-background ratio. The

6 respective network was localized beneath the nucleus (corresponding to z-plane 2 in d). (d) Dual-

7 color labeling of endogenous lamin and vimentin in living cells. $\alpha$-Lamin ${ }^{\text {ATTO655 }}$ and $8 \alpha$-Vimentin ${ }^{\text {ATTO488 }}$ were simultaneously transferred into HeLa Kyoto cells. $3 \mathrm{~h}$ after cell squeezing, 9 spatially distinct labeling of endogenous lamin (red) and vimentin (green) was visualized by 10 multiplexed imaging. Magnification (box, top) of the $\alpha$-Vimentin ${ }^{\text {ATTO488 }}$ stained vimentin network 11 showed the three-dimensional organization around the nucleus. The respective confocal z-planes 12 are indicated in the scheme. (e) Reconstructed dSTORM image of endogenous lamin specifically 13 labeled with $\alpha$-Lamin ${ }^{\mathrm{ATTO}}{ }^{\mathrm{F} 55} \mathrm{Fb}$ (5 $\mathrm{h}$ after squeezing). The sensitive and high degree of Fb labeling 14 allowed for visualization of the native nuclear envelope with nanometer precision. (f) Comparison 15 of $d$ STORM analysis with the averaged fluorescence intensity. Cross section of the intensity 16 profiles (along dashed line in e) displayed the enhanced resolution by dSTROM. Images were 17 taken by CLSM (a-d) with the Airy detector (except for d) or dSTORM (e). Dashed lines indicate 18 the cell border. Scale bars: $5 \mu \mathrm{m}$. 


\section{SUPPLEMENTAL FIGURES}

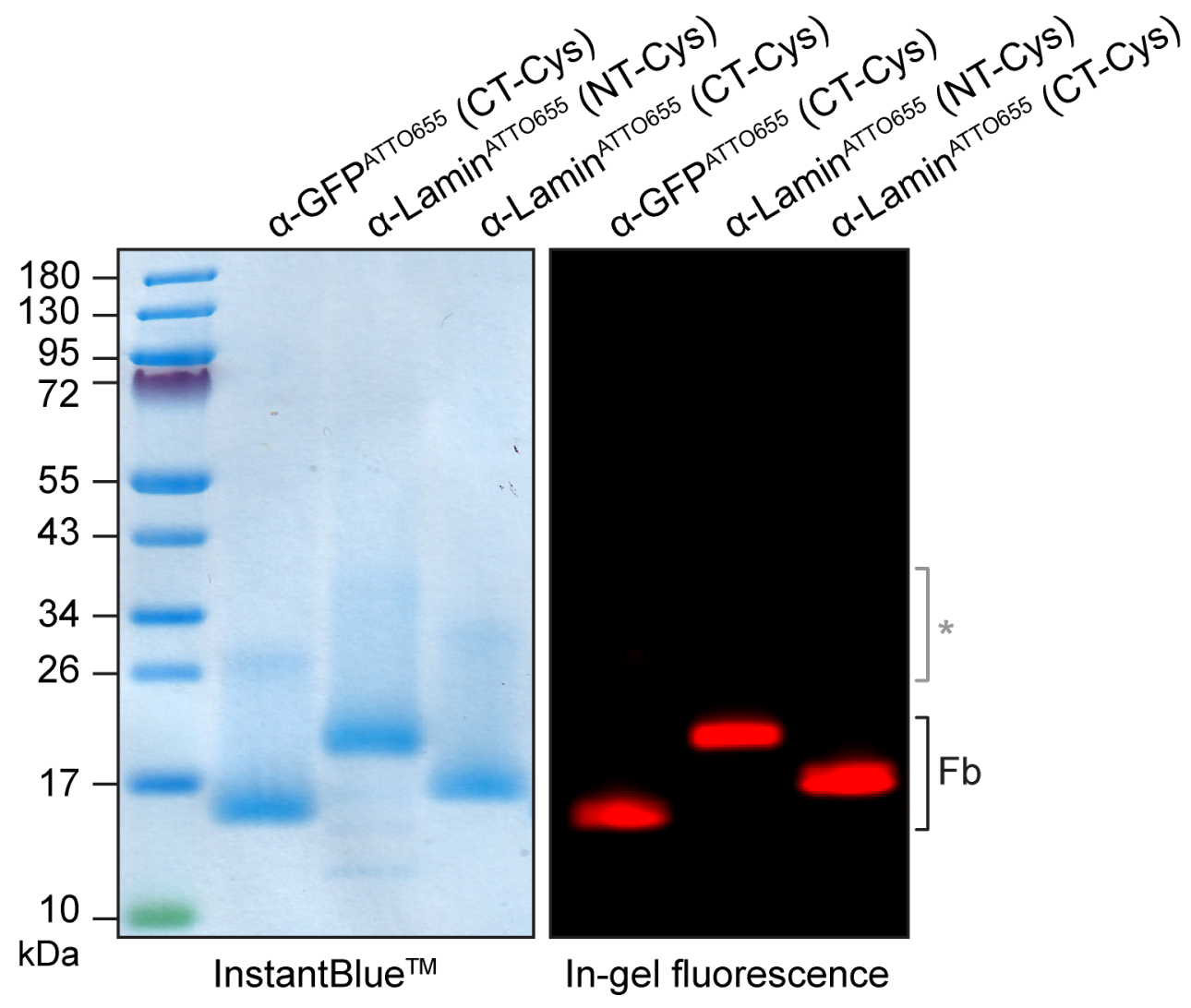

3 Figure 1 - Figure Supplement 1 I Site-specific fluorescent labeling of nanobodies. Single

4 engineered surface cysteines of nanobodies were modified with maleimide ATTO655. SDS-PAGE

5 analysis showed covalent labeling of the engineered Fbs. Detection was performed by

6 InstantBlue ${ }^{\mathrm{TM}}$ staining and by in-gel fluorescence with $\lambda_{\text {ex/em }} 630 / 670 \mathrm{~nm}$. A minor portion of $\mathrm{Fb}$

7 dimers (*) was observed. The higher molecular weight of $\alpha$-Lamin (NT-Cys) is due to an elongated

$8 \quad$ linker between $\mathrm{Nb}$ and $\mathrm{C}$-terminal $\mathrm{His}_{6}$-tag. 


\section{a}

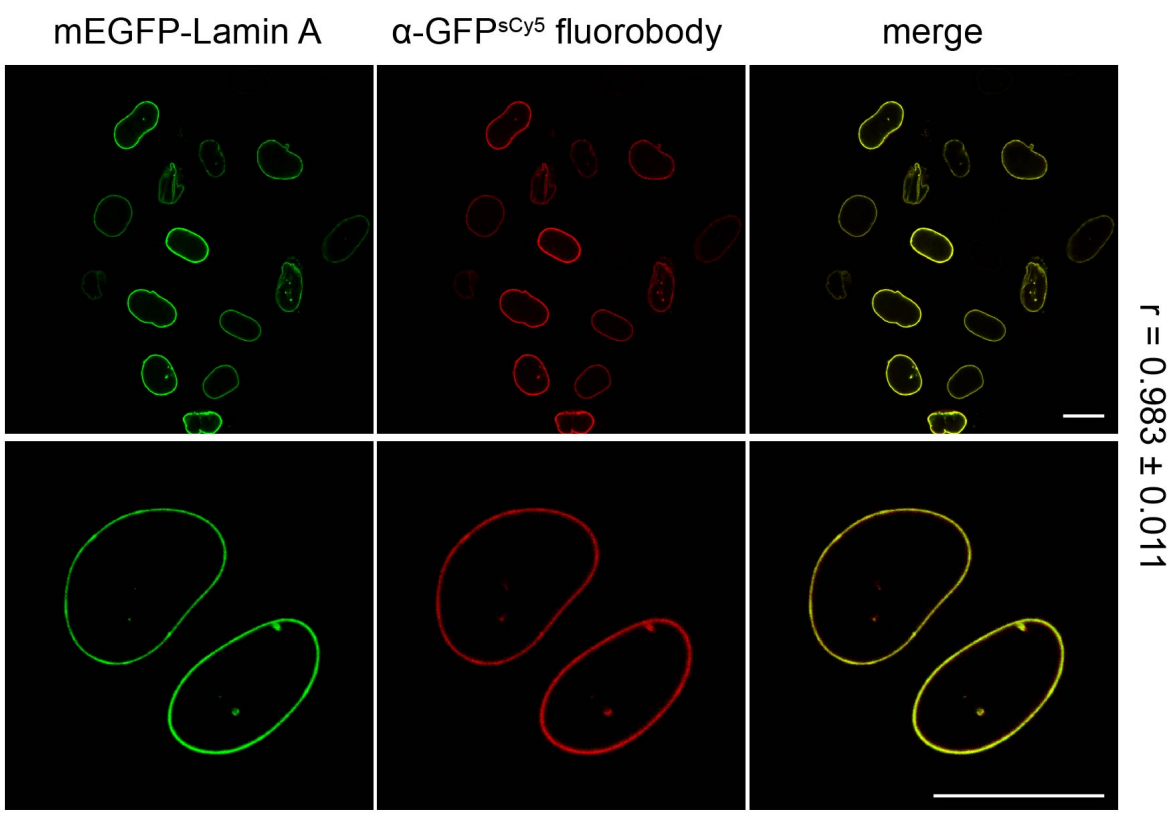

b

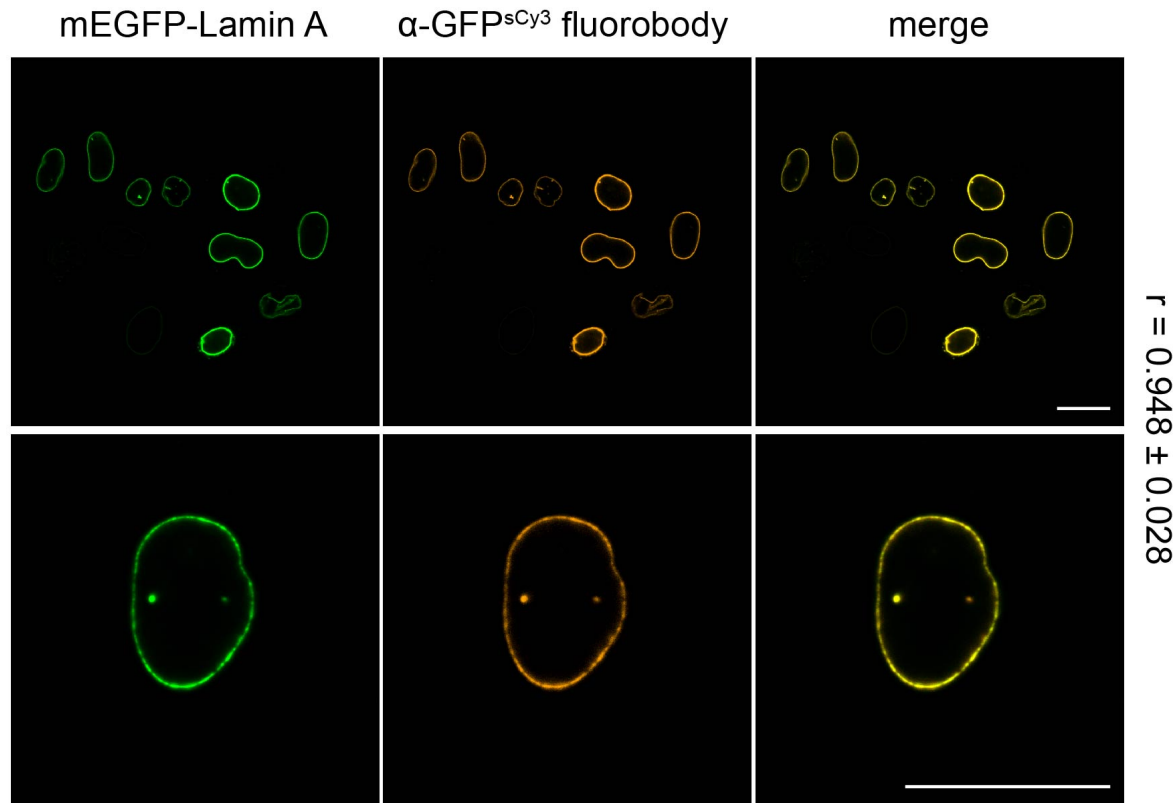

2 Figure 1 - Figure Supplement 2 I Specific labeling of mEGFP-Lamin A with $\alpha-G F P^{\text {sCy3 }}$ and

$3 \quad \boldsymbol{\alpha}-G P^{\text {sCy5 }}$ fluorobodies. After fixation and permeabilization, HeLa Kyoto cells expressing

4 mEGFP-Lamin A were labeled with $100 \mathrm{nM}$ of $\alpha-\mathrm{GFP}^{\mathrm{sCy} 5} \mathrm{Fb}(\mathbf{a})$ or $\alpha-\mathrm{GFP}^{\mathrm{sCy} 3} \mathrm{Fb}(\mathbf{b})$ and analyzed

5 via CLSM. mEGFP-Lamin A was specifically labeled by the Fbs with very low background and the

6 staining intensity correlated with the POI's expression level. Scale bars: $20 \mu \mathrm{m}$. 


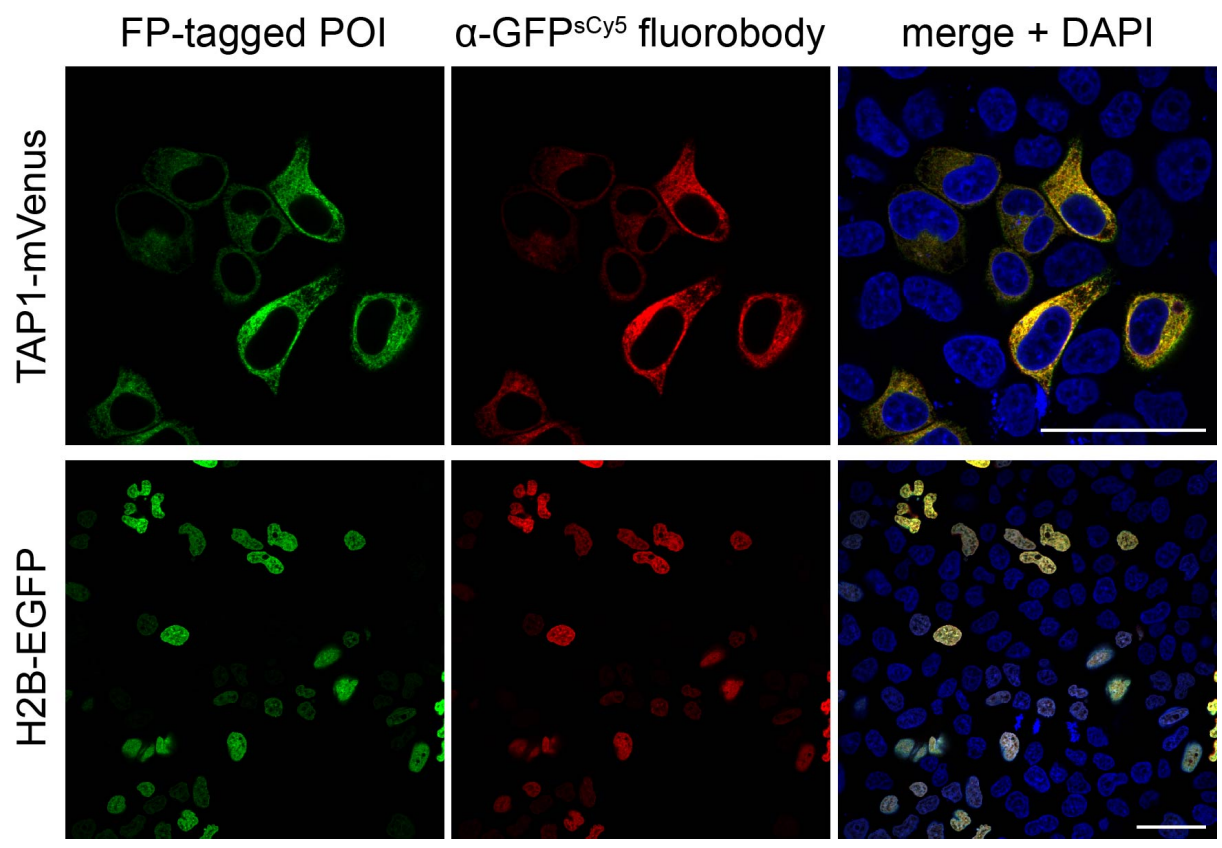

2 Figure 1 - Figure Supplement 3 I Correlation of the target protein expression level with the

3 labeling density of the $\alpha$-GFP fluorobody. After fixation and permeabilization, HeLa Kyoto cells

4 expressing TAP1-mVenus or H2B-EGFP were stained with $\alpha-\mathrm{GFP}^{\mathrm{sCy} 5} \mathrm{Fb}(50 \mathrm{nM})$. The labeling

5 intensity highly correlates with the expression level of both FP-fused POIs, reflecting stoichiometric

$6 \quad$ target decoration. Imaging was performed by CLSM. Scale bars: $50 \mu \mathrm{m}$. 
a

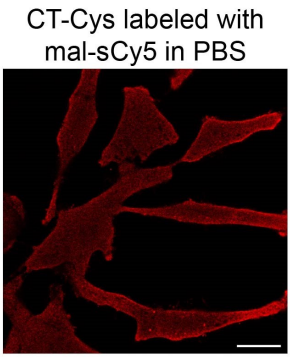

b

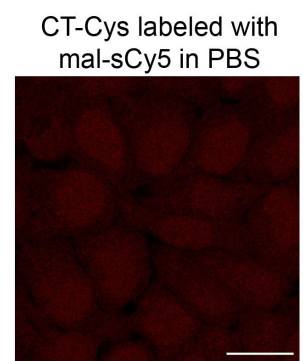

NT-Cys labeled with mal-sCy5 in PBS

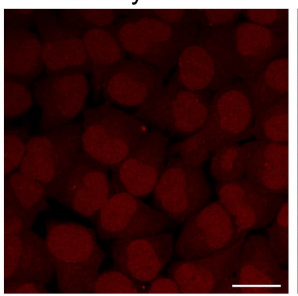

Lys labeled with

NHS-Alexa647

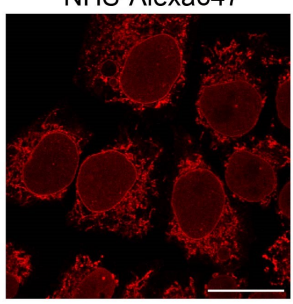

CT-Cys labeled with mal-sCy5 in MLB

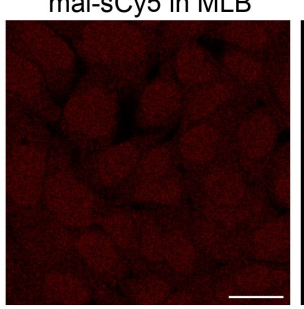

NT-Cys labeled with mal-sCy5 in MLB

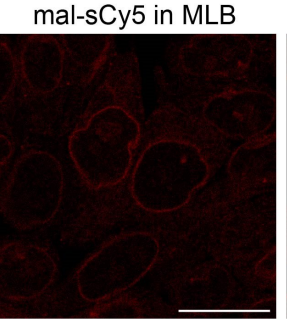

CT-Cys labeled with mal-sCy5 in MLB

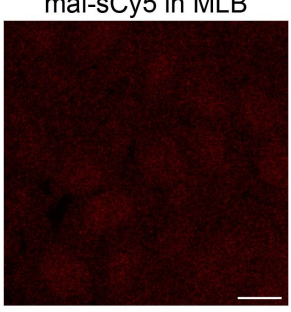

S9C labeled with mal-sCy5 in MLB

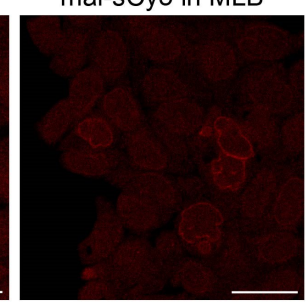

NT-Cys labeled with mal-ATTO655 in MLB

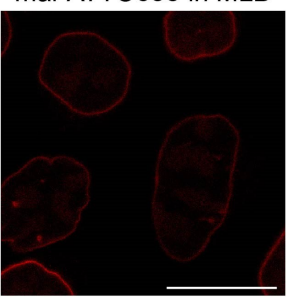

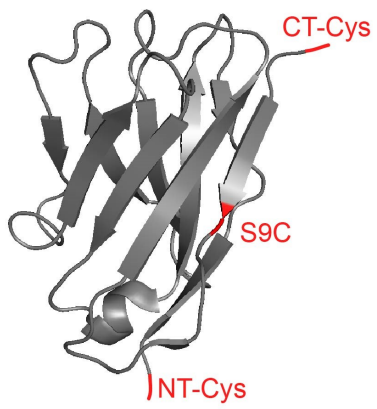

Figure 1 - Figure Supplement 4 I Labeling screen of differently modified $\alpha$-Lamin

fluorobodies. The $\alpha$-Lamin nanobody was engineered with an extra single cysteine at three different positions, e.g. N-terminally (NT-Cys), C-terminally (CT-Cys), or at position 9 (S9C). After production in E. coli BL21/DE3 (a) or E. coli T7 SHuffle Express (b) and affinity purification, the engineered nanobodies were modified with maleimide sCy5 or ATTO655. Stochastic labeling via lysines exposed on the nanobody surface was conducted with NHS Alexa647. The specificity of these Fbs was evaluated by staining of HeLa Kyoto cells (500 nM) after fixation and permeabilization. Fb target specificity was analyzed by CLSM. For the CT-Cys labeled, S9C labeled and Lys labeled Fbs a severe lack in target specificity was observed. After production in E. coli T7 SHuffle Express and subsequent modification of the $\mathrm{N}$-terminal cysteine in maleimide labeling buffer (MLB), specific tracing of endogenous lamin was achieved with greatly enhanced signal-to-background ratio. This Fb format was used for all further experiments. Scale bars: $20 \mu \mathrm{m}$. 

aCC-BY 4.0 International license.

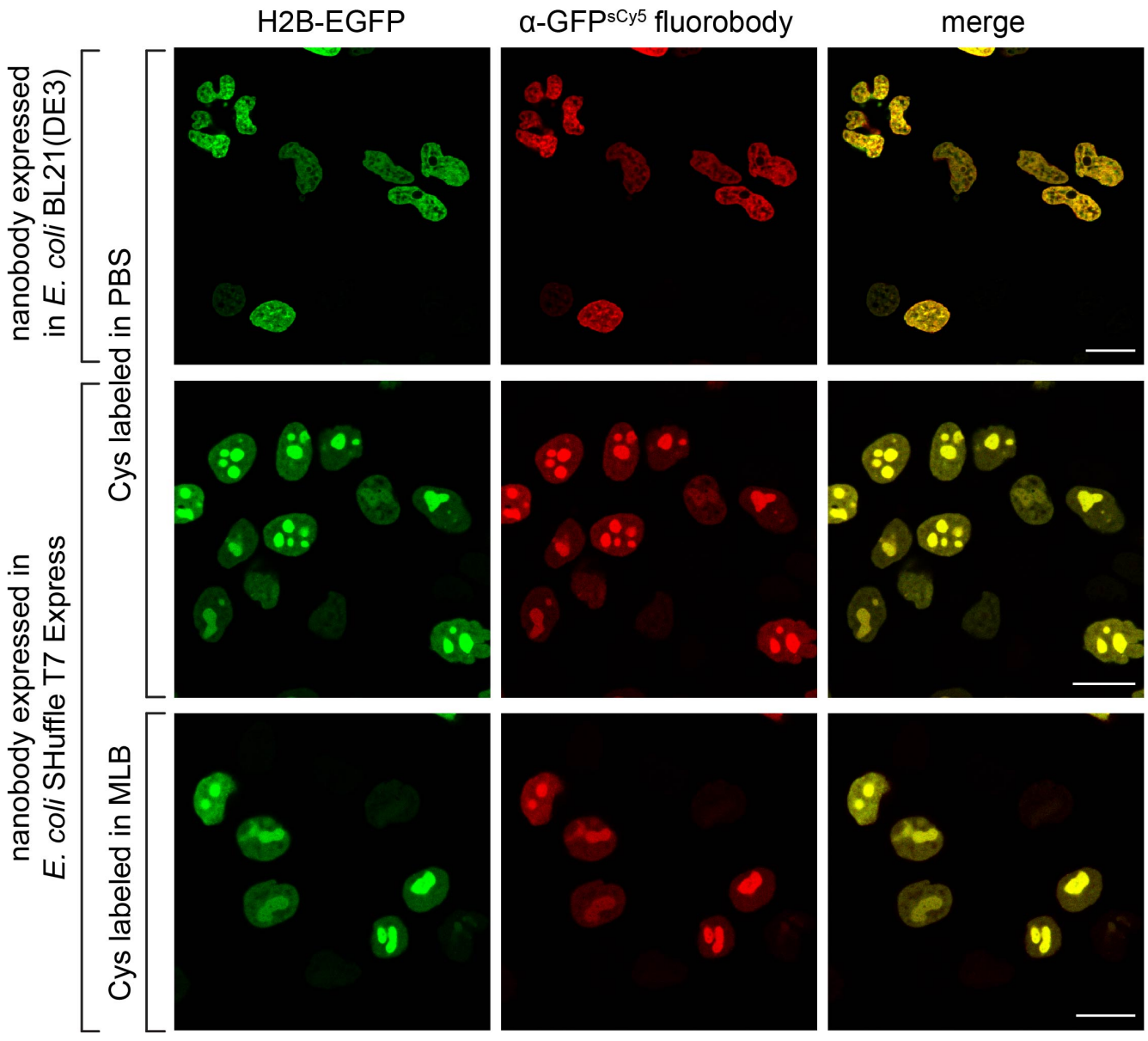

2 Figure 1 - Figure Supplement 5 I Labeling screen of differently prepared $\alpha$-GFP

3 fluorobodies. The $\alpha-\mathrm{GFP}^{\mathrm{sCy} 5} \mathrm{Fb}$ with an N-terminal cysteine was produced in two different $E$. coli

4 strains (BL21/DE3 or T7 SHuffle Express), and modified with maleimide sCy5. a-GFP binding

5 screen was performed against H2B-EGFP (green) for all tested Fb preparation conditions. Fixed

6 and permeabilized HeLa Kyoto cells were stained with $\alpha-\mathrm{GFP}^{\mathrm{sCy} 5} \mathrm{Fb}(100 \mathrm{nM}$, red). All

7 combinations for $\mathrm{Fb}$ generation resulted in specific labeling of EGFP-tagged H2B (merge) by the

8 engineered $\alpha$-GFP with low background labeling. Analysis was performed by CLSM. Scale bars:

$9 \quad 20 \mu \mathrm{m}$. 


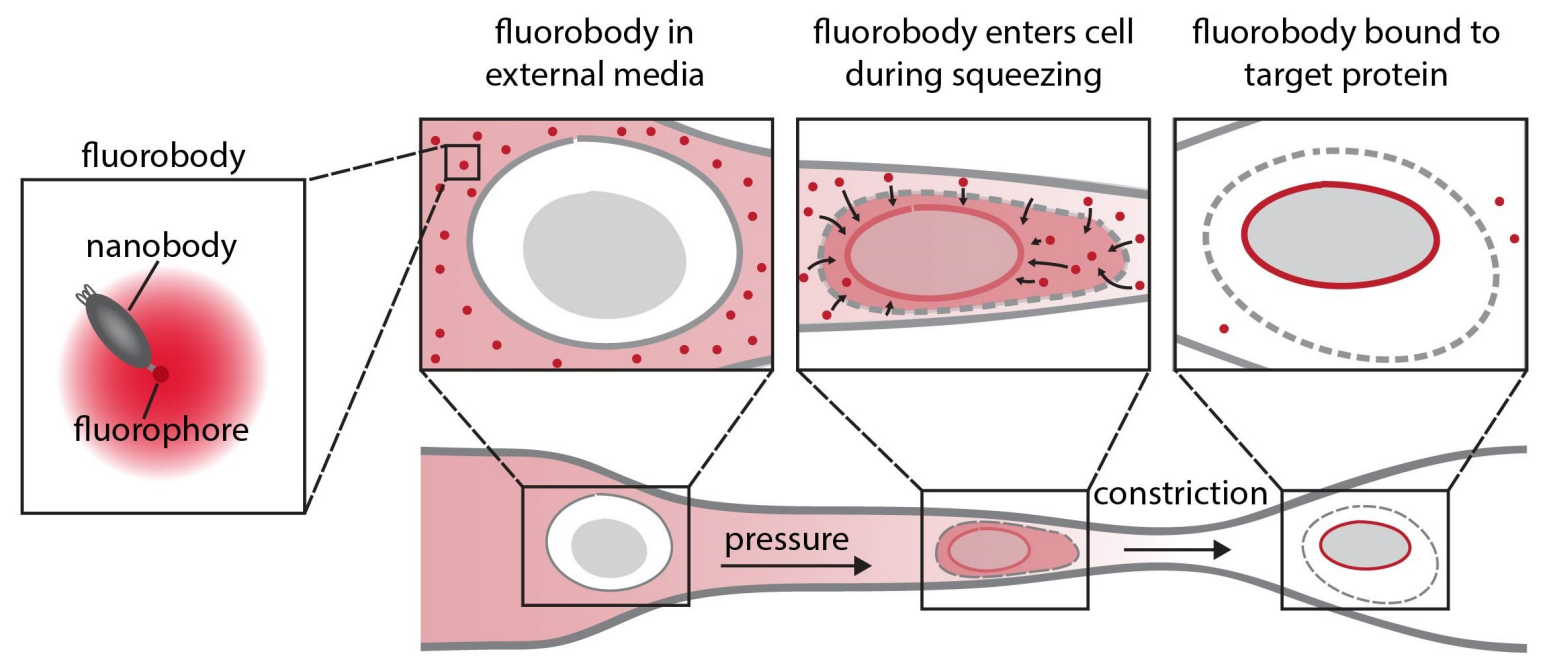

2 Figure 2 - Supplement Figure 1 I Fluorobody transfer into living cells by microfluidic cell

3 squeezing. Cells are pressed through constrictions, which are $\sim 30 \%$ smaller than cell diameter.

4 Shear forces cause the formation of transient holes in the plasma membrane. By passive diffusion

5 (middle), site-specifically labeled Fbs enter the cytosol and bind to their target (right, binding to

6 nuclear envelope is exemplarily illustrated). The induced holes reseal within $\sim 5 \mathrm{~min}$. 


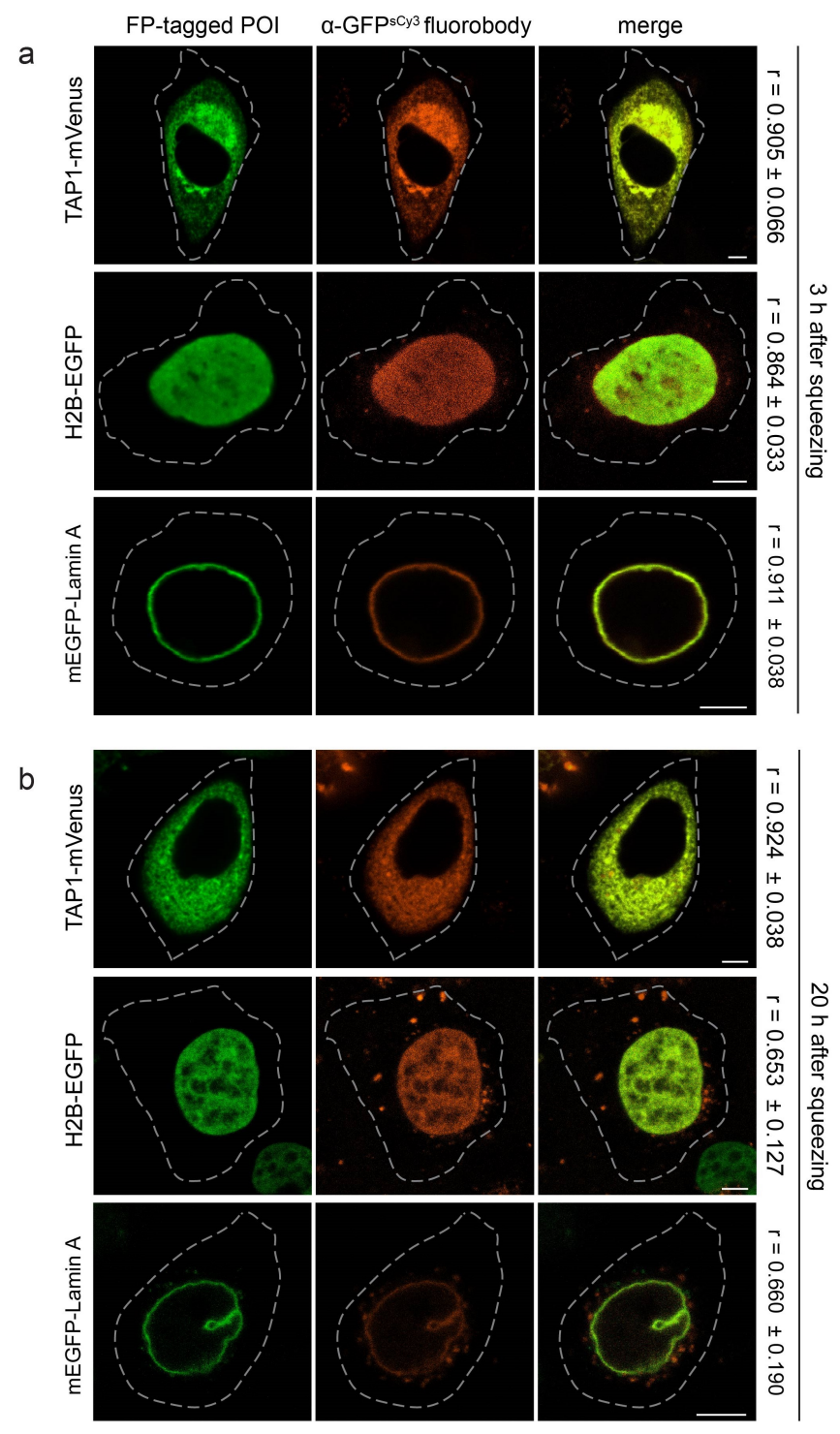

2 Figure 2 - Figure Supplement 2 I Live-cell labeling of FP-tagged targets via the $\alpha$-GFP ${ }^{\text {sCy3 }}$

3 fluorobody. $\alpha-\mathrm{GFP}^{\mathrm{sCy} 3} \mathrm{Fb}(200 \mathrm{nM})$ was transferred into HeLa Kyoto cells transiently transfected

4 with TAP1-mVenus, H2B-EGFP, or mEGFP-Lamin A, respectively. Cells were imaged $3 \mathrm{~h}$ (a) and

$520 \mathrm{~h} \mathrm{(b)}$ after cell squeezing by CLSM. For all tested subcellular localizations of the FP-tagged

$6 \mathrm{POI}$, the molecular specificity of the Fb was persistent for a prolonged period of time $(20 \mathrm{~h})$. After

$720 \mathrm{~h}$, background originating as cytosolic punctae was detected. This observation is supported by

8 a decreased Pearson's coefficient ( $r$, right), calculated from 8-10 individual cells. Dashed lines

$9 \quad$ indicate the cell border. Scale bars: $5 \mu \mathrm{m}$. 


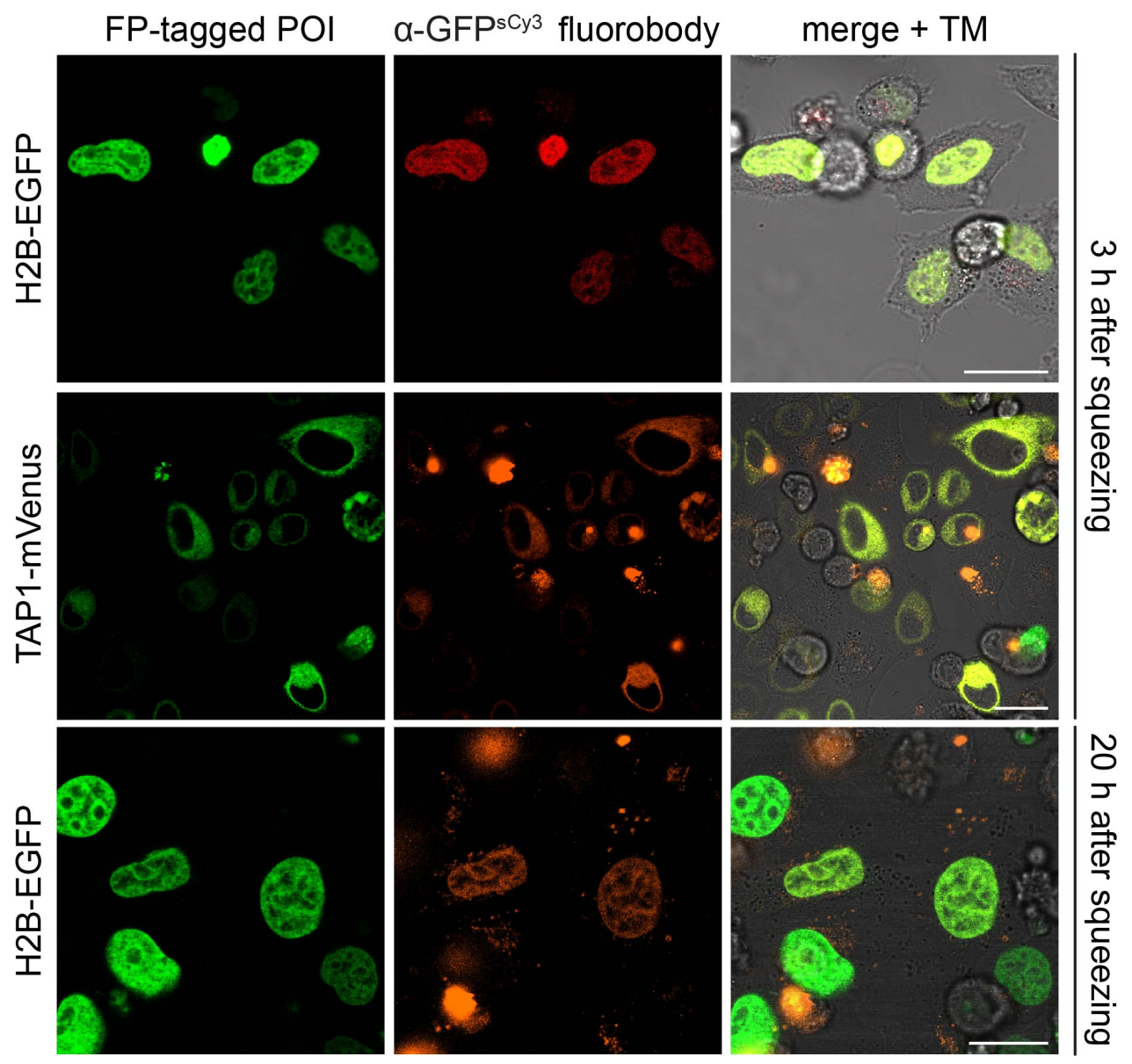

2 Figure 2 - Figure Supplement 3 I High fluorescence intensity correlation between target or H2B-EGFP (green) were squeezed in the presence of $\alpha-\mathrm{GFP}^{\mathrm{sCy} 3} \mathrm{Fb}$ (200 nM, orange). Imaging via CLSM was performed $3 \mathrm{~h}$ and $20 \mathrm{~h}$ after squeezing. Low background and high co-localization between the $\mathrm{Fb}$ and $\mathrm{POI}$ was observed in multiple cells with varying expression level. Incipient

7 punctual background was detectable after $20 \mathrm{~h}$ (bottom). Scale bars: $20 \mu \mathrm{m}$. 


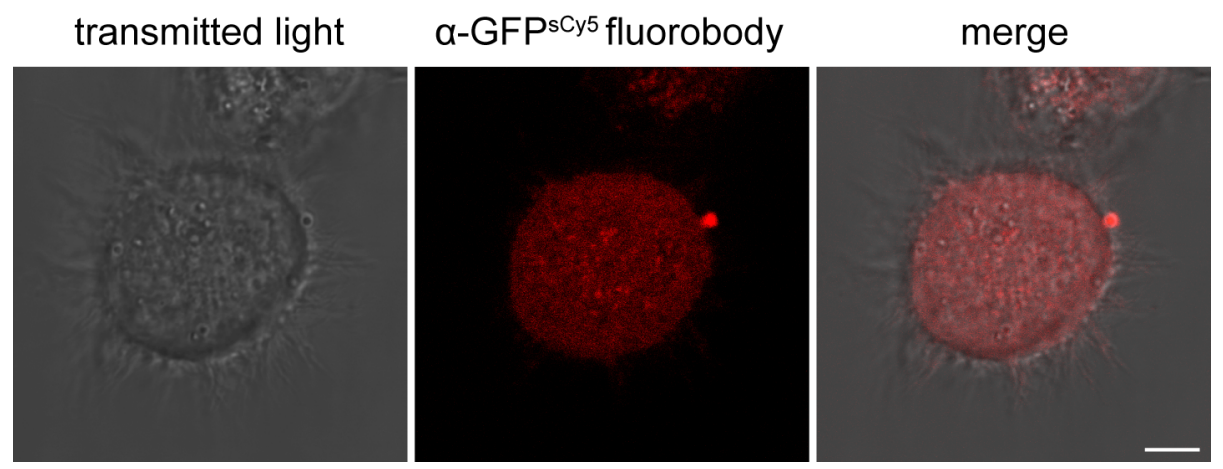

2 Figure 2 - Figure Supplement 4 I Cytosolic distribution of $\alpha$-GFP ${ }^{\text {sCy5 }}$ fluorobody in

3 untransfected cells. $\alpha-G F P^{\mathrm{sCy} 5} \mathrm{Fb}(100 \mathrm{nM})$ was delivered into HeLa Kyoto cells via cell

4 squeezing. A cytosolic distribution of the $\mathrm{Fb}$ (red) was detected $1 \mathrm{~h}$ after squeezing. In absence

5 of FP-tagged proteins, no distinct cellular localization of $\alpha-G F P^{\mathrm{sCy} 5}$ Fbs was observed, confirming

6 the molecular specificity of the $\alpha-\mathrm{GFP}^{\mathrm{sCy} 5} \mathrm{Fb}$ for antigen tracking. Imaging was performed by

7 CLSM. Scale bar: $5 \mu \mathrm{m}$. 


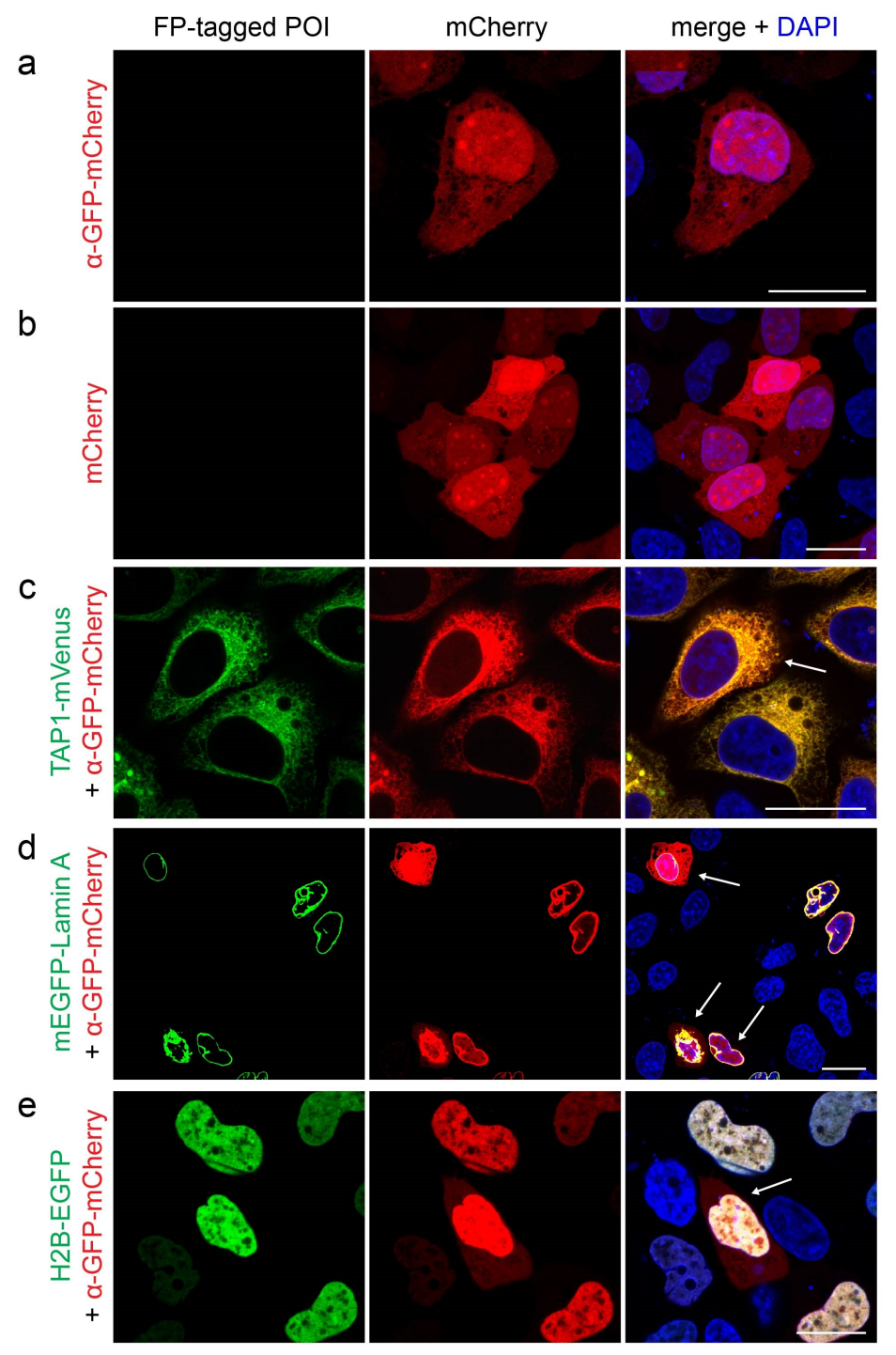

2 Figure 2 - Figure Supplement 5 I Transient expression of $\alpha$-GFP-mCherry chromobody in

3 mammalian cells. (a) In the absence of the target protein, the a-GFP-mCherry nanobody was

4 homogenously distributed within the cytosol and nucleus, similar to mCherry alone (b). (c-e) HeLa

5 Kyoto cells were transiently co-transfected with an a-GFP-mCherry chromobody (red) and a FP-

6 tagged POI (TAP1-mVenus, mEGFP-Lamin A or H2B-EGFP; green). Increased chromobody expression levels resulted in a high background of unbound nanobody, distributed in the cytosol

8 and nucleus (indicated by arrows). Before CLSM imaging, HeLa Kyoto cells were chemically

$9 \quad$ arrested with $4 \%$ formaldehyde and additionally stained with DAPI (blue). Scale bars: $20 \mu \mathrm{m}$. 

aCC-BY 4.0 International license.

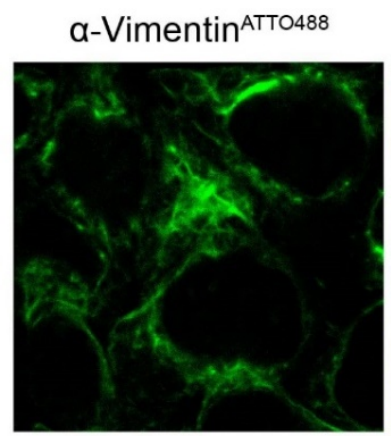

merge with DAPI
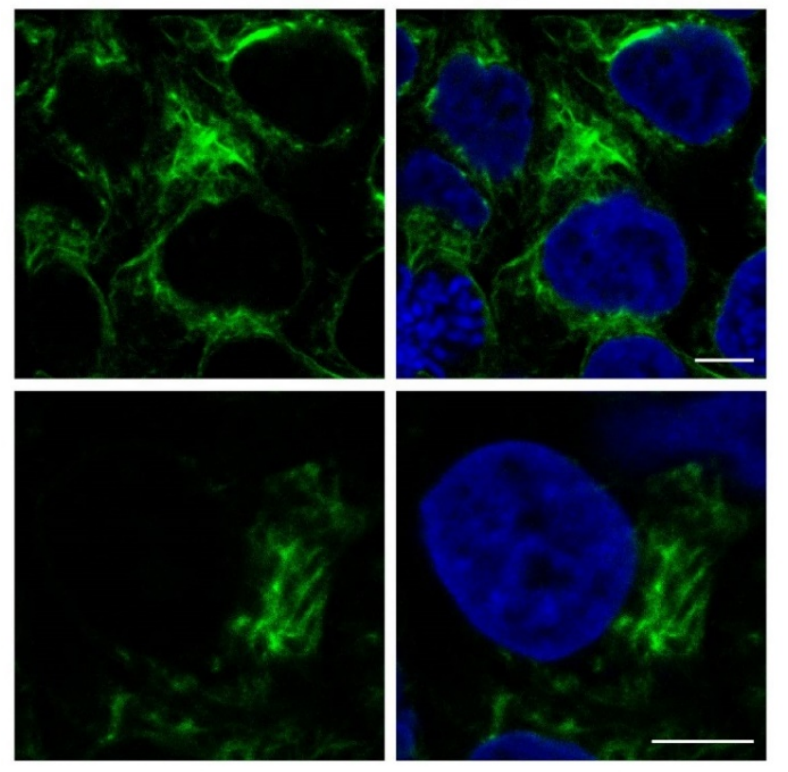

2 Figure 3 - Figure Supplement 1 I Vimentin staining in fixed cells. HeLa Kyoto cells were fixed,

3 permeabilized and stained with $\alpha$-Vimentin ${ }^{\text {ATTO488 }}(20 \mu \mathrm{g} / \mathrm{ml}$, green) and DAPI (blue). Confocal

4 imaging showed specific labeling of endogenous filamentous vimentin. Scale bar: $5 \mu \mathrm{m}$. 

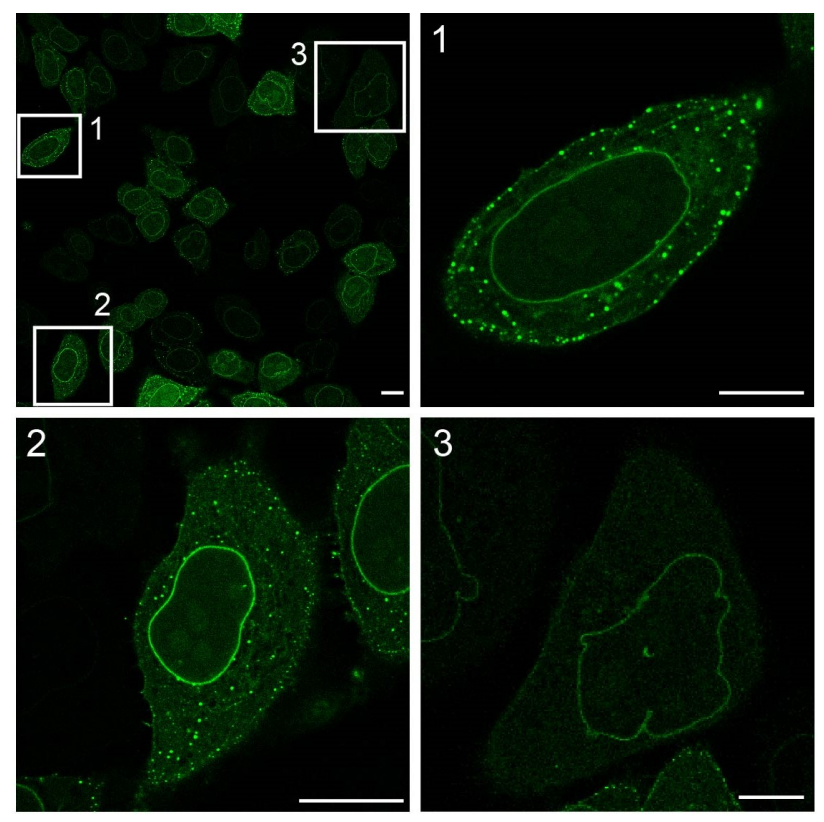

2 Figure 3 - Figure Supplement 2 I Transient expression of $\alpha$-Lamin-EGFP chromobody in

3 HeLa Kyoto cells entails background of free nanobody. Cells transiently transfected with

4 a-Lamin-EGFP were fixed with $4 \%$ formaldehyde $24 \mathrm{~h}$ after transfection. Specific decoration of

5 endogenous lamin at the nuclear envelope was visualized by CLSM. The variability of the

6 chromobody expression level was accompanied by a high background, originating as cytosolic

7 punctae. Images 1-3 are magnifications of individual cells as indicated by white boxes in the upper

$8 \quad$ left image. Scale bars: $10 \mu \mathrm{m}$. 

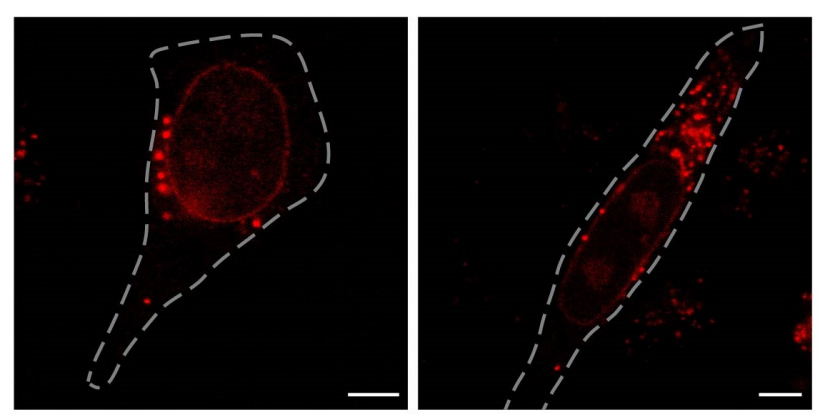

3 Figure 3 - Figure Supplement 3 I Persistence of endogenous lamin targeting by Fb in living

4 cells. The $\alpha$-Lamin ${ }^{\mathrm{sCy} 5} \mathrm{Fb}(500 \mathrm{nM})$ was transferred into native HeLa Kyoto cells via cell

5 squeezing. Specific decoration of the endogenous nuclear lamina by the Fb was still observed

$620 \mathrm{~h}$ after squeezing by CLSM. An enriched punctual background was detected compared to $3 \mathrm{~h}$

7 post squeezing (see Fig. 3a). Dashed lines indicate the cell border. Scale bars: $5 \mu \mathrm{m}$. 
a

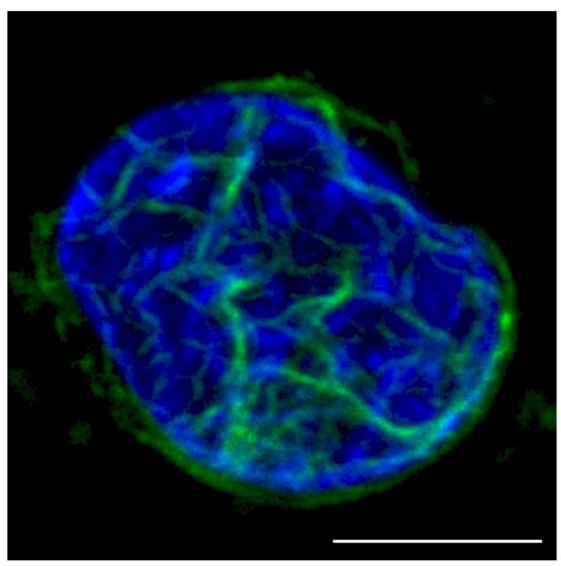

b

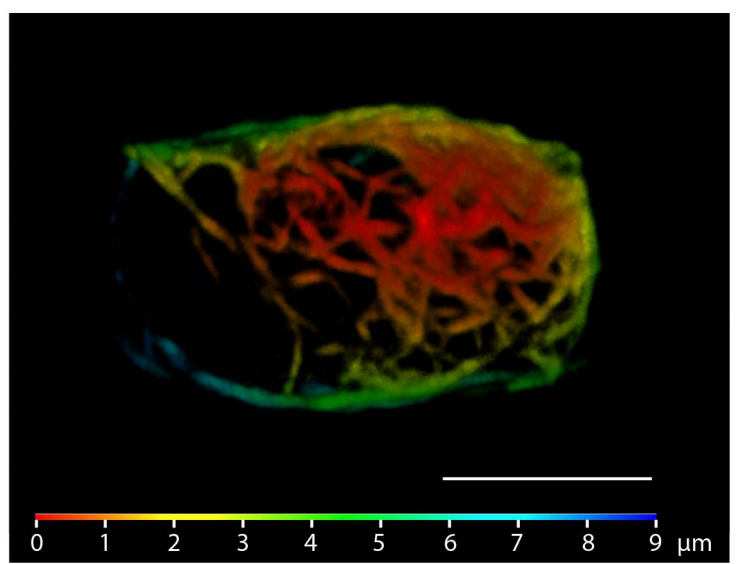

2 Figure 3 - Figure Supplement 4 I 3D reconstruction of endogenous vimentin network. After

3 intracellular transfer of $\alpha$-Vimentin ${ }^{\text {ATTO488 }}(50 \mu \mathrm{g} / \mathrm{ml})$, z-stacks were recorded by confocal imaging

$4 \quad$ using the Airy scan detector (SR mode, Zeiss). (a) Imaging was performed $3 \mathrm{~h}$ after squeezing

5 with additional HOECHST staining (blue). The 3D reconstruction of the obtained z-stack showed

6 the complex network of endogenous vimentin (green), organized around nucleus. (b) 3D

7 topographic map of the endogenous vimentin meshwork. The series of z-stacks was recorded

$820 \mathrm{~h}$ after cell squeezing and reconstructed. The rainbow-color code indicates the depth. Distance

9 between stacks: $\sim 200 \mathrm{~nm}$, respectively. Scale bars: $5 \mu \mathrm{m}$. 


\section{a}

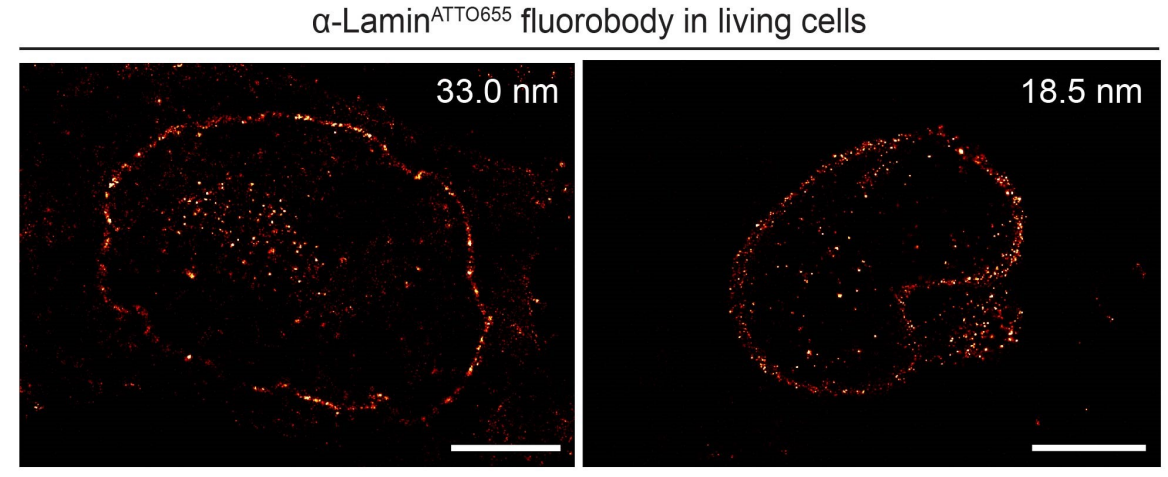

a-LaminATT0655 fluorobody in fixed cells
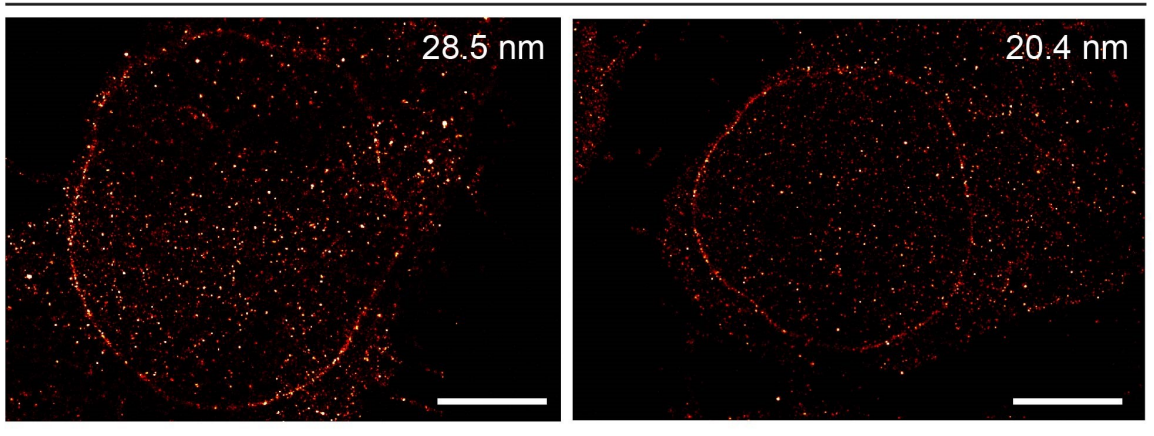

b
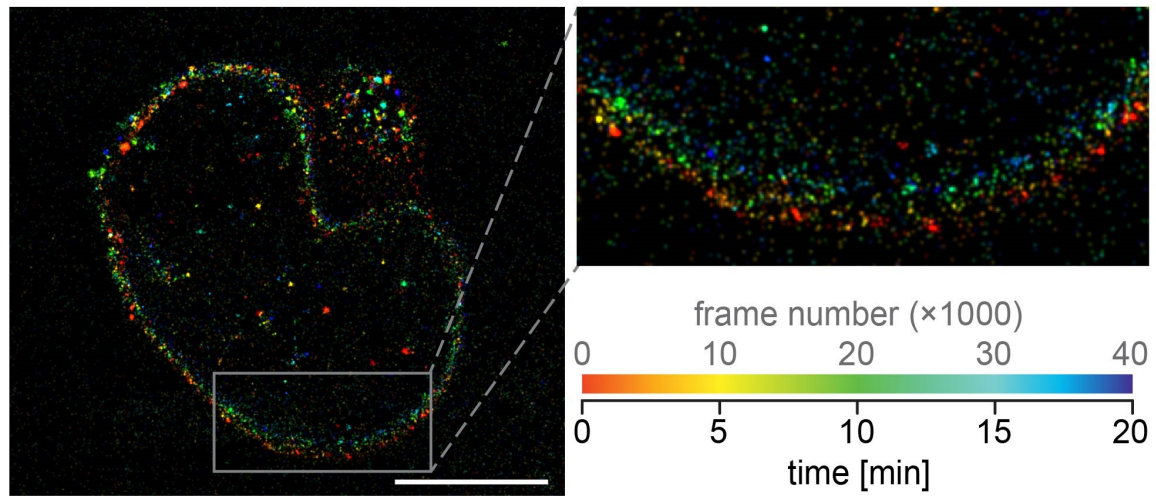

Figure 3 - Figure Supplement 5 I Super-resolution imaging of endogenous lamin in fixed

3 and living cells. HeLa Kyoto cells were either squeezed with $\alpha$-Lamin ${ }^{\mathrm{ATTO}}{ }^{\mathrm{F} 5} \mathrm{Fb}$ (500 nM) for live-

4 cell imaging or fixed, permeabilized and stained with $\alpha$-Lamin ${ }^{\text {ATTO655 }}$ Fb (100 nM). (a) dSTORM

5 analysis revealed a similar average localization precision in living (23.4 $\pm 8.3 \mathrm{~nm}$, top) and fixed

$6 \quad(23.2 \pm 4.6 \mathrm{~nm}$, bottom $)$ cells. The localization precision determined for each image is given in the

7 top right corner. Mean and standard deviation were calculated from three images. (b) Cell

8 dynamics during live-cell super-resolution microscopy can impede imaging. Frame numbers are

9 color-coded. Scale bar: $5 \mu \mathrm{m}$. 
1 Supplementary Video 1 I 3D reconstruction of the endogenous nuclear lamina. After fixation

2 and permeabilization, endogenous lamin of a HeLa Kyoto cell was visualized with an

$3 \alpha$-Lamin ${ }^{\mathrm{ATTO} 055} \mathrm{Fb}(100 \mathrm{nM}) .53$ individual stacks were recorded by confocal imaging using the

4 Airy scan detector (Zeiss, distance between stacks $\sim 200 \mathrm{~nm}$ ). 3D reconstruction showed a dense

5 decoration of the complete nuclear lamina by the $\mathrm{Fb}$, accompanied with a high signal-to-

6 background ratio. Several intranuclear structures of endogenous lamin were observed. 